\title{
Syllable is a synchronization mechanism that makes human speech possible
}

\author{
Yi Xu
}

Department of Speech, Hearing and Phonetic Sciences, Division of Psychology and Language

Sciences, University College London, UK. Chandler House 2 Wakefield Street, London

WC1N 1PF, United Kingdom, yi.xu@ucl.ac.uk

\begin{abstract}
Speech is a highly skilled motor activity that shares a core problem with other motor skills: how to reduce the massive degrees of freedom (DOF) to the extent that the central nervous control and learning of complex motor movements become possible. It is hypothesized in this paper that a key solution to the DOF problem is to eliminate most of the temporal degrees of freedom by synchronizing concurrent movements, and that this is done in speech through the syllablea mechanism that synchronizes consonantal, vocalic and laryngeal gestures. Under this hypothesis, gestures are articulatory movements toward underlying targets; the onsets and offsets of the gestures are synchronized at the syllable edges, although more so at syllable onset than at the offset; and the realization of the synchronization is facilitated by sensorimotor feedback, especially tactile feedback, during consonant closures. This synchronization theory of the syllable also offers a comprehensive account of coarticulation, as it explicates how various coarticulation-related phenomena, including coarticulation resistance, locus, locus equation, diphone etc., are byproducts of syllable formation. It also provides a theoretical basis for understanding how suprasegmental events such as tone, intonation, phonation, etc. are aligned to segmental events in speech. It may also have implications for understanding vocal learning, speech disorders and motor control in general.
\end{abstract}


Although nearly everyone can identify syllables, almost nobody can define them.

— Ladefoged (1982, p. 220)

The human motor apparatus ... comprises more than 200 bones, 110 joints and over 600 muscles, each one of which either spans one, two or even three joints. While the degrees of freedom are already vast on the biomechanical level of description, their number becomes dazzling when going into neural space.

- Huys (2010, p. 70)

\section{Degrees of freedom and motor synchrony}

One of the fundamental problems in motor control, as first recognized by Nikolai Bernstein (Bernstein, 1967), is that of too many degrees of freedom (DOF). That is, most motor movements involve multiple body structures, so that it would be immensely difficult for the central nervous system to control them separately. Speech, also as a motor skill, faces the same problem. To say a simple syllable like [ma], for example, multiple articulatory gestures need to be made concurrently: closing the glottis and increasing the lung pressure to generate voice, closing the lips and lowering the velum to produce the nasal sound [m], lowering the jaw, lowering and retracting the tongue body to produce the vowel [a] (Stevens, 1998). And, if the [ma] is said with a lexical tone in a language like Mandarin, the vocal folds have to be adjusted to raise or lower $F_{0}$, often more than once, within the same syllable $(\mathrm{Xu}, 1997)$. All of these need to be completed in less than one fifth of a second (Tiffany, 1980). In fact, it would be hard to imagine how any motor action involving more than one body structure and/or muscle would be possible without a solution to this DOF problem, and how speech would be possible without a means to reduce DOF to the extent that multiple articulators and the muscles driving them can be effectively controlled.

This DOF problem, which may emerge at any level of neuromotor control, is also known as the Bernstein problem, and there have been many proposals in the motor control literature on how to resolve it (Latash, Scholz \& Schöner, 2007). Bernstein's own proposal is that motor redundancy can be minimized by freezing many of the degrees of freedom by organizing a group of muscles into a functional unit called synergy, "such that a central control signal jointly and proportionally activates all muscles in the synergy" (Latash et al., 2007:278). A further idea is that groups of muscles may form coordinative structures that act together to perform a single action (Easton, 1972; Turvey, 1977). Coordinative structure has also been adopted in some theories of speech production (Fowler et al., 1980; Saltzman \& Munhall, 1989). In particular, it is applied in the task dynamic (TD) model of speech articulation (Saltzman \& Munhall, 1989) which forms the computational basis of articulatory phonology (AP) (Browman \& Goldstein, 1992a). In this TD/AP framework, within each coordinative structure, the articulatory gestures are assumed to be autonomous entities that are temporally overlapped with each other. To coordinate these overlapping gestures, a coupled oscillator model of timing planning is then 
applied (Goldstein, Byrd \& Saltzman, 2006; Nam, Goldstein \& Saltzman, 2009; Saltzman et al., 2008). In this model, each gesture is associated with an internal planning oscillator responsible for controlling the temporal pattern of its movement. In this conceptualization, however, not only the multiple gestures, but also their relative timing is separately controlled. This does not seem like a solution for the DOF problem, as there is at least no conceptual reduction of degrees of freedom.

The coupled oscillator model has been used to account for various synchronization phenomena in motor movements (Haken, Kelso, \& Bunz, 1985; Kay et al., 1987; Semjen \& Ivry, 2001), because motor synchrony appears to resemble the well-known physical entrainment (Huygens, 1893). Entrainment is a physical phenomenon whereby two oscillating systems with similar natural frequencies, e.g., two pendulum clocks, gradually fall into synchrony when they are connected through some mechanical link, such as being hung on the same beam (Huygens, 1893; Pikovsky, 2001). If coupled oscillation does not resolve the DOF problem as pointed out above, however, we may wonder whether entrainment is the right analogy for motor synchrony. Indeed, a careful comparison reveals that motor synchrony differs from entrainment in a number of critical ways, as listed in Table 1. First, motor synchrony can occur in bi-manual actions with no repeating cycles (Kelso, Southard \& Goodman, 1979). Such non-repeating synchrony, by definition, would be irrelevant to entrainment. But it is highly relevant for monosyllabic words spoken in isolation. Likewise, in speaking in unison - a skill surprisingly natural to most people without much practice, speakers can easily synchronize their reading aloud of the same text (Cummins, Li \& Wang, 2013). The non-periodic articulatory movements in speaking in unison cannot be accounted for by theories that use periodicity as the basis of explaining synchronization (Cummins, 2011).

Table 1. Motor synchrony versus entrainment.

\begin{tabular}{lll}
\hline Property & Motor synchrony & Entrainment \\
\hline Synchrony in a single cycle? & Yes & N/A \\
\hline \hline Speed of achieving synchrony & Immediate (1-2 cycles) & Many cycles \\
\hline Similarity in natural frequency? & No & Yes \\
\hline In-synch out-synch undulation? & No & Yes \\
\hline Under central/shared control? & Yes & No \\
\hline
\end{tabular}

Second, in entrainment, it takes many cycles for two oscillators to reach synchrony. In motor synchrony, the shift from $180^{\circ}$ (anti-phase) to $0^{\circ}$ (in-phase) occurs in only $1-2$ cycles (Kelso, 1984; Kelso, Tuller \& Harris, 1983; Mechsner et al., 2001; Schmidt, Carello \& Turvey, 1990), which is virtually instantaneous. In a system of coupled oscillators, the fastest phase shift Haken et al. (1985) simulated with an oscillation model takes 5-6 cycles to complete. A gradual shift across 5-6 cycles also means that in some of those cycles the phase relation is neither $180^{\circ}$ nor $0^{\circ}$, which is exactly what has been repeatedly shown to be impossible in motor synchrony (Kelso, Saltzman \& Tuller, 1986; Mechsner et al., 2001; Schmidt et al., 1990). Furthermore, in coupled oscillation, each oscillator has to have its own initial phase condition. For speech, one would naturally ask, where do the initial phase conditions come from in the first place? 
Third, as shown in the third and fourth rows of Table 1, entrainment requires that the synchronized oscillators are similar in their natural frequencies, and even after reaching synchrony, they may go out of phase again (Adler, 1946; Bennet et al., 2002). Neither high similarity in frequency nor phase instability is characteristic of motor synchrony (Kelso et al., 1979; Mechsner et al., 2001).

Finally, probably the most fundamental difference is that in entrainment, the systems being synchronized are independent of each other, with no central control, and the synchrony is achieved passively through physical links between the involved systems (Pikovsky, 2001). Motor synchrony, in contrast, occurs between movements that are under a common central control, or in the case of synchrony between two individuals, under a shared control maintained through sensory monitoring (Schmidt et al., 1990). The central control, as well as the sensory monitoring that makes it possible, are clearly lacking in physical entrainment.

The Bernstein problem therefore is unlikely to be solved by a coordinative structure constructed as a system of coupled oscillators (Goldstein et al., 2006; Nam et al., 2009; Saltzman et al., 2008). To start with, there is no explicit scheme to reduce degrees of freedom. Rather, each of the assumed planning oscillator has to have its own natural frequency unrelated to the natural frequency of the associated gesture, and its own initial phase condition, which results in at least two additional degrees of freedom. Furthermore, entrainment is used to model both abrupt shifts from VC to CV in accelerating repetitive syllable sequences (Kelso, Tuller \& Harris, 1983), and the planning of intergestural coordination postulated to occur before the onset of each and every syllable (Goldstein et al., 2006; Nam, Goldstein \& Saltzman, 2009; Saltzman et al., 2008). It is especially problematic that it takes at least several cycles to complete a phase shift or to stabilize the phase relation during planning when they are computationally modeled as an entrainment process (Haken et al., 1985).

A viable alternative solution therefore needs to explicate how degrees of freedom can be effectively reduced. The solution considered in this paper is that for speech, the syllable serves exactly this function. This solution, however, suggests a theory of the syllable that differs from all past syllable theories. And it also touches on the long-standing issue of coarticulation. The following section briefly reviews the state of the art for both syllable and coarticulation.

\section{Syllable and coarticulation}

That speech utterances are made up of syllables may seem obvious. Most, if not all, early writing systems (Sumerian, Linear B, Akkadian cuneiform, Chinese, Mayan, etc.) started as syllabaries, in which the written symbols represent syllables (or sometimes morae) rather than consonants and vowels (DeFrancis, 1989; Gnanadesikan, 2011; Liberman et al., 1974). It is also much easier for anyone, including non-experts, to count the number of syllables in a word than the number of segments in a syllable (Fox \& Routh, 1975; Liberman et al., 1974; ShattuckHufnagel, 2011). The syllable is also known to play many important roles in speech. It is the unit that carries stress and accent (Bolinger, 1961; de Jong, 2004; Pierrehumbert, 1980), rhythm (Barbosa \& Bailly, 1994; Cummins \& Port, 1998; Nolan \& Asu, 2009) and tone (Abramson, 1978; Chao, 1968). It is the domain of applying many phonological rules (Blevins, 2001; Hooper, 1972). It is also critical for the perceptual segmentation of the speech signal (Bertoncini \& Mehler, 1981; Content, Kearns \& Frauenfelder, 2001; Cutler et al., 1986). However, neither 
our intuition about it nor its own usefulness has been sufficient to avert doubts about the syllable. After examining eight lines of traditional evidence in support of the syllable as a representation unit in speech production, Shattuck-Hufnagel (2011) found none of them unequivocal. The lack of clear evidence has led to scepticisms about its existence (Kohler, 1966) or universality (Labrune, 2012). Similar reservations have been expressed by Gimson (1970), Steriade (1999) and Blevins (2003). A major reason that doubts about the syllable cannot be fully dissolved is that its nature has remained vague. In particular, no theory has been able to address some of the hardest questions about the syllable:

(1) Why are there syllables?

(2) Do syllables have clear phonetic boundaries?

(3) Do segments have definitive syllable affiliations?

\subsection{Why are there syllables?}

Some theories have taken syllable as the basic unit of speech, e.g., Stetson's motor phonetics (Stetson, 1951) and Fujimura's (1994) C/D model. But they have offered no explicit proposal as to why syllables are obligatory at the articulatory level. In MacNeilage's (1998) frame/content theory, the syllable is suggested to have evolved from the oscillation of the jaw in such movements as chewing, sucking and licking. However, the ability to oscillate the jaw is shared by virtually all mammals, yet not even our closest relatives, i.e., chimpanzees and gorillas, have developed syllable-based speech (Fitch, 2010; Pinker, 1995). Thus being able to oscillate the jaw does not seem to inevitably lead to an ability to articulate syllables. Something extra must be involved.

It has also been proposed that the syllable is a unit of stored motor programs (Dell, 1988; Levelt, Roelofs \& Meyer, 1999). But the proposal is questioned for its inability to explain cases of resyllabification or the lack thereof (Shattuck-Hufnagel, 2011). More importantly, even if stored syllable-sized motor programming is shown to exist, it cannot explain why the unit has to have the form of syllable. It thus remains an open question as to whether the syllable, with its own unique characteristics, is indispensable, i.e., serving a function that is so vital that speech would be impossible without it.

\subsection{Are there clear boundaries to the syllable?}

Given an utterance like the one shown in Figure 1, it may seem that some of the syllables are well separated by the alternation of consonants and vowels whose spectral patterns show clear boundaries (Jakobson, Fant \& Halle, 1951). However, the syllable boundaries are much less clear-cut in the case of /wei/. Because it begins with a glide /w/, it is hard to determine when the preceding syllable ends and the next one starts. Even more difficult are cases where a word starts with a vowel, as in the English words like artist, article, articulate, arbitrary. When they are preceded by words ending in a vowel, as in new artist, my article, to articulate, or fairly arbitrary, there would be continuous formant movements across the word (hence syllable) boundaries (unless when spoken very carefully so that the syllable starts with a glottal stop). 
The same problem would be seen in cases of word internal syllables, like in hiatus, appreciate, mediocre, etc., where there should presumably be a syllable boundary between /i/ and the following vowel or diphthong, yet all we can see in the spectrogram in most cases are continuous formants between the preceding and following consonants.

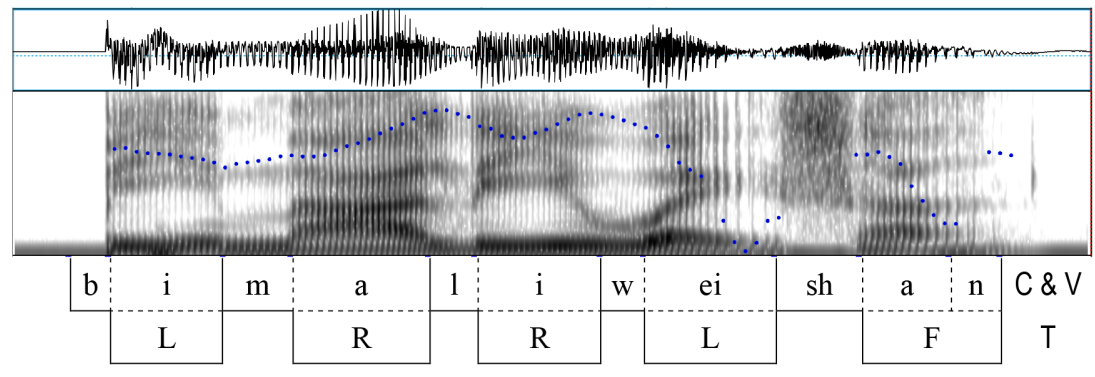

Figure 1. Spectrogram of the Mandarin phrase “比麻黎偽善” /bǐ má lí wěi shàn/ [more hypocritical than $\mathrm{Ma} \mathrm{Li}$, with broad phonetic transcriptions. In both panels, $\mathrm{C}, \mathrm{V}$ and $\mathrm{T}$ stands for consonant, vowel and tone. In a. the segmentation is conventional (Jakobson et al., 1951; Turk, Nakai \& Sugahara, 2006). The segmentation of $/ \mathrm{w} /$ is based on Peterson and Lehiste (1960). In b. the segmentation is based on the synchronization hypothesis.

The difficulty of syllable boundary identification has led to the view that it is simply futile to look for clear-cut boundaries in the speech signal, as argued by Hockett (1955), who likens segments as coloured raw Easter eggs lined up on a belt. After being crushed by a wringer, the heavy smearing makes the edges of the individual eggs unrecognizable. But if we don't know where the boundaries are, how can we be so certain that segments are heavily overlapped with each other (Farnetani \& Recasens, 1997; Kühnert \& Nolan, 1999)? So the fuzziness of the syllable boundaries is directly related to the fuzziness of segmental boundaries, which in turn is related to yet another major conundrum of human speech: coarticulation, as will be discussed in 2.4 .

\subsection{Do segments have definitive syllable affiliations?}

The clarity of syllable boundaries hinges on not only the clarity of segmental boundaries, but also the certainty about where each and every segment should belong in a syllable: onset, offset or between two adjacent syllables, i.e., being ambisyllabic. There have been many theories of syllabification, including the law of initials and law of finals (Vennemann, 1988), the maximal onset theory (Pulgram, 1970; Steriade, 1982), the theory that stressed syllables are maximized (Hoard, 1971, Wells, 1990) and the weight-stress principle (Fudge, 1969; Selkirk, 1982; Wells, 1990). But so far there has been no consensus on even some of the simplest cases. For the word happy, for example, at least four ways of syllabification are possible as summarized by Duanmu (2009): /hæ.pi/, /hæp.i/, /hæpi/ and /hæp.pi/ (where the period stands for syllable boundary and an underscore indicates the segment is ambisyllabic). All these syllabification theories, however, are based on intuition or non-experimental phonological analyses. There are also experimental investigations of syllabification intuition by naïve speakers (Chiosáin, Welby \& Espesser, 2012; Content et al., 2001; Goslin \& Frauenfelder, 2001; Schiller, Meyer \& Levelt, 1997). None of 
the syllabification findings, however, has directly addressed the issue of what syllable boundaries look like in the acoustic signal or in terms of articulatory movements.

\subsection{Syllable and coarticulation}

The problems of syllable boundary and syllable affiliation of segments discussed above are both closely related to another long-standing problem, namely, coarticulation. The term coarticulation, initially "Koartikulation" in German, was coined to refer to articulatory timing around syllable onset (Menzerath \& de Lacerda, 1933). The observation was that "the articulatory movements for the vowel in tokens such as $/ \mathrm{ma} / \mathrm{or} / \mathrm{pu} / \mathrm{began}$ at the same time as the movements for the initial consonant" (Kühnert \& Nolan, 1999:14). The link between syllable and coarticulation is further strengthened by Kozhevnikov and Chistovich (1965), who proposed the notion articulatory syllable, based on the observation that in Russian the lip protrusion of $/ \mathrm{u} /$ begins at the same time as the first consonant in a consonant cluster. According to this notion, the domain of coarticulation is the articulatory syllable, in the sense that all the articulatory actions connected with the articulatory syllable, including the vowel, start at the syllable onset, as long as the consonantal movements do not contradict the articulation of the vowel.

Articulatory syllable, however, has been questioned due to uncertainties over the temporal scope of vowel in a syllable (Gay, 1978; Kent \& Minifie, 1977; Kent \& Moll, 1972; Moll \& Daniloff, 1971). A major reason for the skepticism is the widely reported preparatory activities, particularly the classic finding of Öhman (1966), and the phenomenon of vowel harmony (Clements, 1976), which seem to suggest that the scope of the vowel extends well before the syllable onset. By now, the term coarticulation is generally used to refer to virtually any variability of a segment due to the influence of surrounding segments (Kühnert \& Nolan, 1999).

Perhaps the most detailed account so far of coarticulation as related to the syllable is offered by the TD/AP framework (Goldstein et al., 2006; Nam et al., 2009), as mentioned earlier. In the most recent version of $\mathrm{TD} / \mathrm{AP}$, syllable structure is modeled as emerging from coupled oscillations as internal planners that are in-phase between consonant and vowel gestures at the syllable onset but anti-phase at the syllable offset. The in-phase coupling of CV at the syllable onset is consistent with the notion of articulatory syllable (Kozhevnikov \& Chistovich, 1965), and would account for a large amount of CV coarticulation. However, the TD/AP account of the syllable leaves some core problems unresolved. The most critical is the assumption that each gesture is controlled by a planning oscillator whose frequency and initial phase both need to be specified, making it unclear how DOF can be effectively reduced. Second, the in-phase and anti-phase assumption for syllable onset versus offset is based on empirical observations, but there is no account of why the asymmetry is there in the first place. Finally, as shown in Table 1, it is questionable that coupled oscillation based on physical entrainment is the right model for motor synchrony due to multiple discrepancies between the two kinds of synchrony.

\section{Syllable as a synchronization mechanism}

The hypothesis considered in this paper is that the DOF problem is solved by actively controlled motor synchrony, which, in the case of speech, is achieved through the syllable. The synchronization fixes the relative timing of multiple motor movements so that most of the 
temporal degrees of freedom are eliminated, not only in learning, but also in normal operation (as opposed to Bernstein's proposal that the freezing of DOF is mainly for learning). For speech, the formation of the syllable is also the mechanism underlying coarticulation. The syllable model based on this hypothesis consists of three core mechanisms: target approximation, edge synchronization and tactile anchoring, as sketched in Figure 2. Target approximation (the dashed curves) is the articulatory process of executing phonetic targets, and it is what defines the articulatory gesture. Edge synchronization (the vertical lines) is the mechanism of coordinating multiple gestures that make up a syllable, including consonant, vowel, tone and phonation $^{1}$. And tactile anchoring (not directly represented in Figure 2) is the facilitation of edge synchronization by sensory feedback, mainly through tactile sensation during consonant closures.

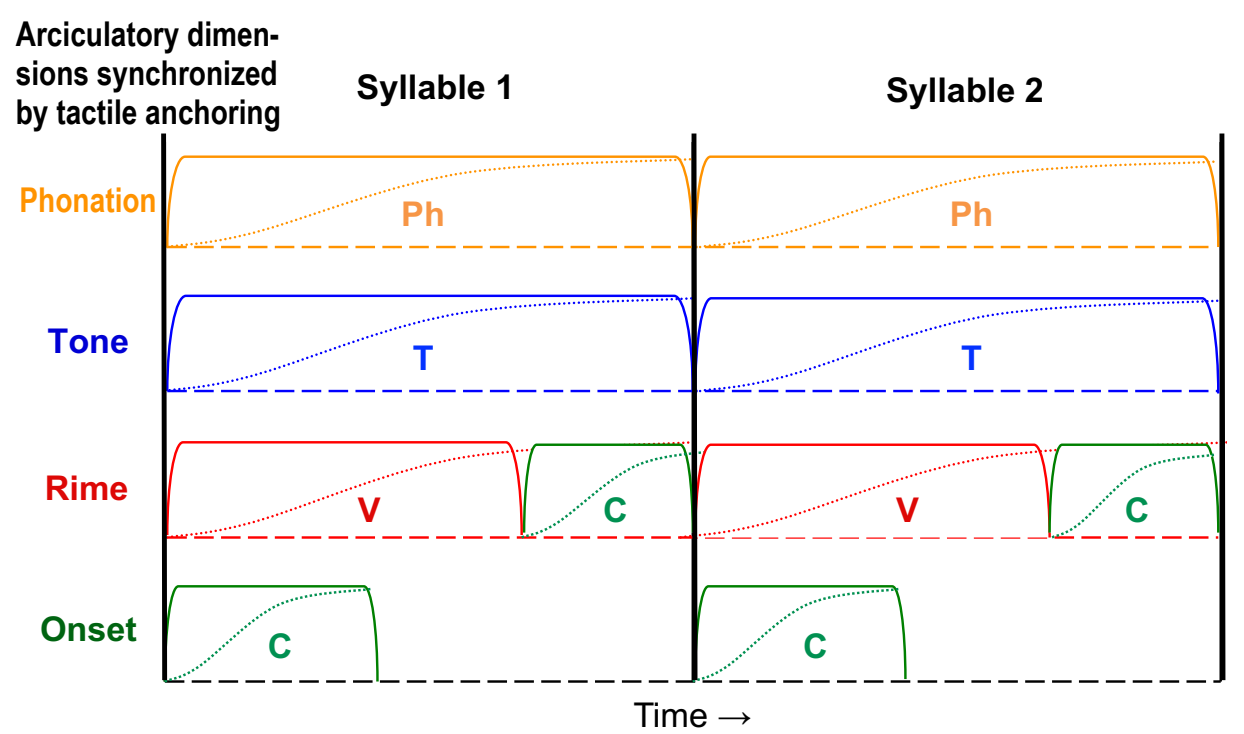

Figure 2. Temporal organization of articulatory dimensions under the synchronization hypothesis. The tiers represent the four articulatory dimensions controlled by the central nervous system. The dotted curves represent asymptotic articulatory approximation of underlying targets (target approximation). The full alignment of the onsets and offsets of the approximation movements represent edge synchronization facilitated by tactile anchoring.

Conceptually, the three mechanisms of the synchronization hypothesis are interlocked as illustrated in Figure 3: Target Approximation is what defines the onsets and offsets of individual movements; movement onsets and offsets (not acoustic landmarks) are what edge synchronization aligns; and tactile anchoring is what ensures the accuracy of synchronization.

\footnotetext{
${ }^{1}$ Here phonation refers to the use of voice quality as an independent dimension to mark lexical contrasts, which is found in some languages (Huffman, 1987; Wayland and Jongman, 2003). It does not refer to phonation properties that accompany consonant manner of articulation.
} 


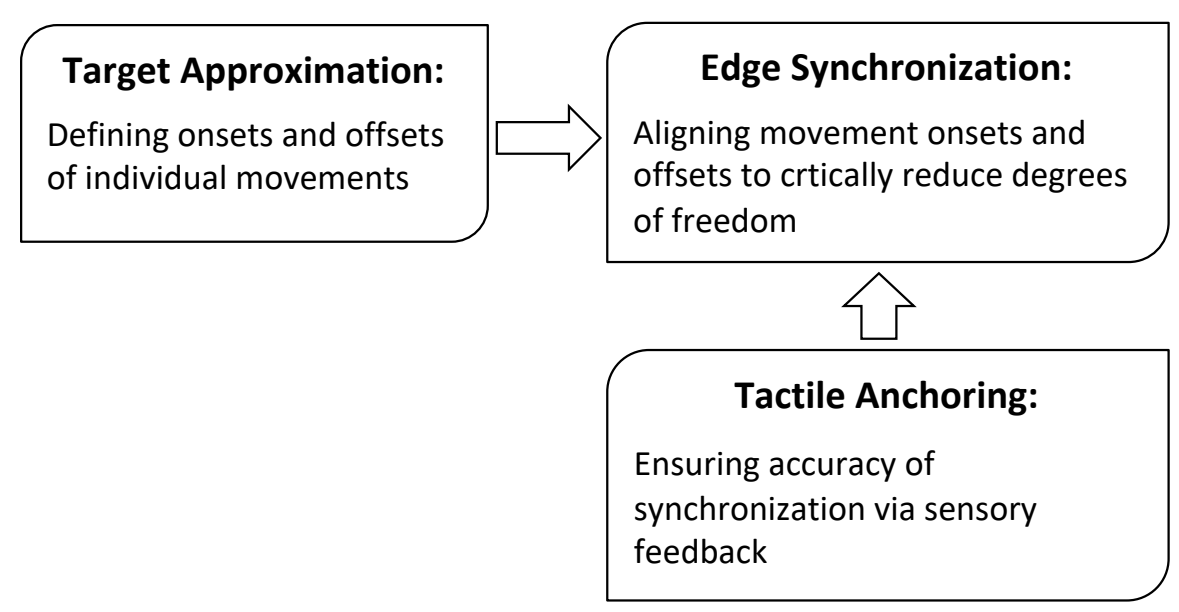

Figure 3. Interdependence of the three mechanisms of the synchronization hypothesis. Target Approximation defines the onsets and offsets of individual movements; edge synchronization aligns movement onsets and offsets (rather than acoustic landmarks); and tactile anchoring provides the sensory feedback that ensures the accuracy of synchronization.

By positing the syllable as a mechanism for solving the DOF problem, the synchronization hypothesis not only offers an account of the syllables that deviates from existing theories, but also provides an account of coarticulation, as will be detailed in 4.1-4.3. In the following sections, each of the three core mechanisms of the synchronization hypothesis will be elaborated, with support from existing literature. Also will be discussed are the similarities and differences between this hypothesis and other models in addressing various specific aspects of the syllable.

\subsection{Targets and target approximation}

The notion of target approximation goes back at least as far as Lindblom (1963), who suggests that underlying phonetic targets are often only partially realized due to time constraint. Similar ideas are shared by a number of models proposed since Lindblom (1963), in particular, the Fujisaki model of intonation (Fujisaki, 1983), and the TD/AP framework for segmental articulation (Browman \& Goldstein, 1992a; Saltzman \& Munhall, 1989). The version of target approximation presented here ( $\mathrm{Xu} \&$ Wang, 2001), as schematized in Figure 4, was independently developed based on empirical data on contextual tonal variations (Xu, 1997, 1998, 1999, 2001).

In this model, each movement is a process of approaching an underlying target (dashed lines in Figure 4) within an extrinsically designated temporal interval. Each target approximation movement is controlled by three parameters: target position, target slope (underlying velocity) and target strength. Adjacent target approximations are contiguous without overlap, shifting abruptly from one to the next at the interval boundary. The resulting surface contour (solid curve in Figure 4) is nevertheless smooth and continuous due to the transfer of dynamic states at the boundary. Despite similarities with other models, there are five key properties that are unique to the target approximation model as presented in Figure 4: 


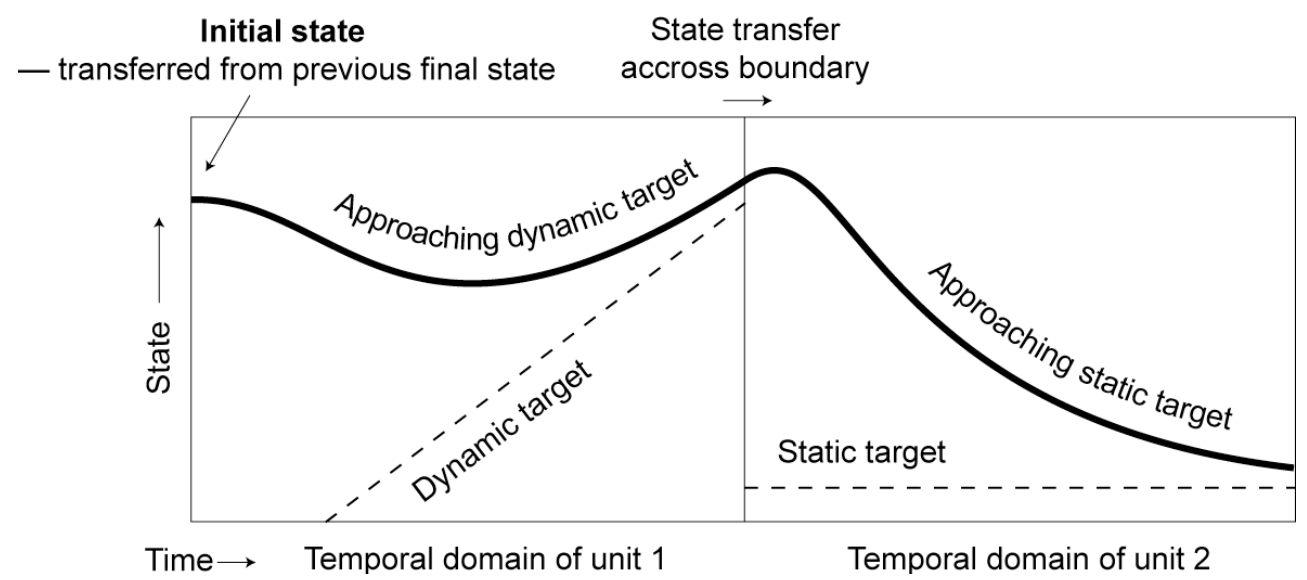

Figure 4. The target approximation model. A schematic illustration of hypothetical phonetic targets (dashed lines) and their surface realization (solid curve). The three vertical lines represent the boundaries of the two consecutive target intervals. The level dashed line on the right represents a static target, and the oblique dashed line on the left represents a dynamic target. In both intervals, the targets are asymptotically approximated. Adapted from the original version for tone and intonation (Xu \& Wang, 2001).

1. Surface acoustic forms result from asymptotic approximation of a single sequence of underlying targets rather than from superposition of multiple underlying contours (Fujisaki, 1983; Bailly \& Holm, 2005; van Santen et al., 2005).

2. Targets are approximated sequentially, with neither overlap of adjacent movements (Browman \& Goldstein, 1989), nor gaps in between (Fujisaki, 1983; Pierrehumbert, 1980), unless there is a silent pause. The lack of gaps also means that there are no temporal intervals (except pauses) without targets.

3. Targets can be intrinsically dynamic, i.e., with underlying slopes of various degrees. No other model, to our knowledge, has incorporated dynamic targets. ${ }^{2}$

4. Every target also has a strength specification, which determines the rate at which the articulatory goal is approached. Target strength (or stiffness) is treated in other models as either mostly fixed (Fujisaki, 1983; Browman \& Goldstein, 1989) or a means of controlling duration (Byrd \& Saltzman, 2003).

5. Target duration is not predominantly determined by the time needed to reach the target, or intrinsic timing (Fowler, 1980; Saltzman \& Munhall, 1989), but by functional factors such as lexical tone, lexical stress, focus and boundary marking (Xu, 2009).

The target approximation model has been quantified in the form of qTA (Prom-on, Xu \& Thipakorn, 2009). The present paper will not focus on the quantitative aspect of the model, but

\footnotetext{
${ }^{2}$ See 3.1.5 for critical differences between underlying velocity and surface velocity. The former is a property of the target, which can be either static or dynamic, while the latter is the consequence of executing the target. Some models, like TD and Fujisaki model, specify stiffness of the target gesture, which indirectly specifies surface velocity. But they have no specifications for underlying velocity. So a fully achieved target in those models can only generate an asymptote to a static articulatory state.
} 
some of the graphics (Figures 5) are generated with qTA. Also, although the target approximation model was initially developed for tones and intonation, its relevance for segments has also been demonstrated (Cheng \& Xu, 2013). Birkholz et al. (2011) have developed a higher-order version of the target approximation model for an articulatory synthesizer, which has been successfully implemented in articulatory synthesis for English, Thai and German (Prom-on et al., 2013, 2014; Xu et al., 2019).

In the following sections, the main properties of target approximation will be elaborated and evidence from the literature, wherever available, will be presented.

\subsubsection{Asymptotic approximation}

Among the clearest evidence of asymptotic approximation are the $\mathrm{F}_{0}$ trajectories of lexical tones found in connected speech $(\mathrm{Xu}, 1997,1999)$. As shown in Figure 5, the $\mathrm{F}_{0}$ of the tone in the $3^{\text {rd }}$ syllable in each plot starts at very different heights depending on the tone of the $2^{\text {nd }}$ syllable. Yet all the trajectories quickly accelerate away from the initial states, and converge, within the $3^{\text {rd }}$ syllable, to a linear configuration that reflects the tone's underlying targets: high-level, falling, and rising, respectively. Similar asymptotic approximation has also been observed for vowels (Moon \& Lindblom, 1994).

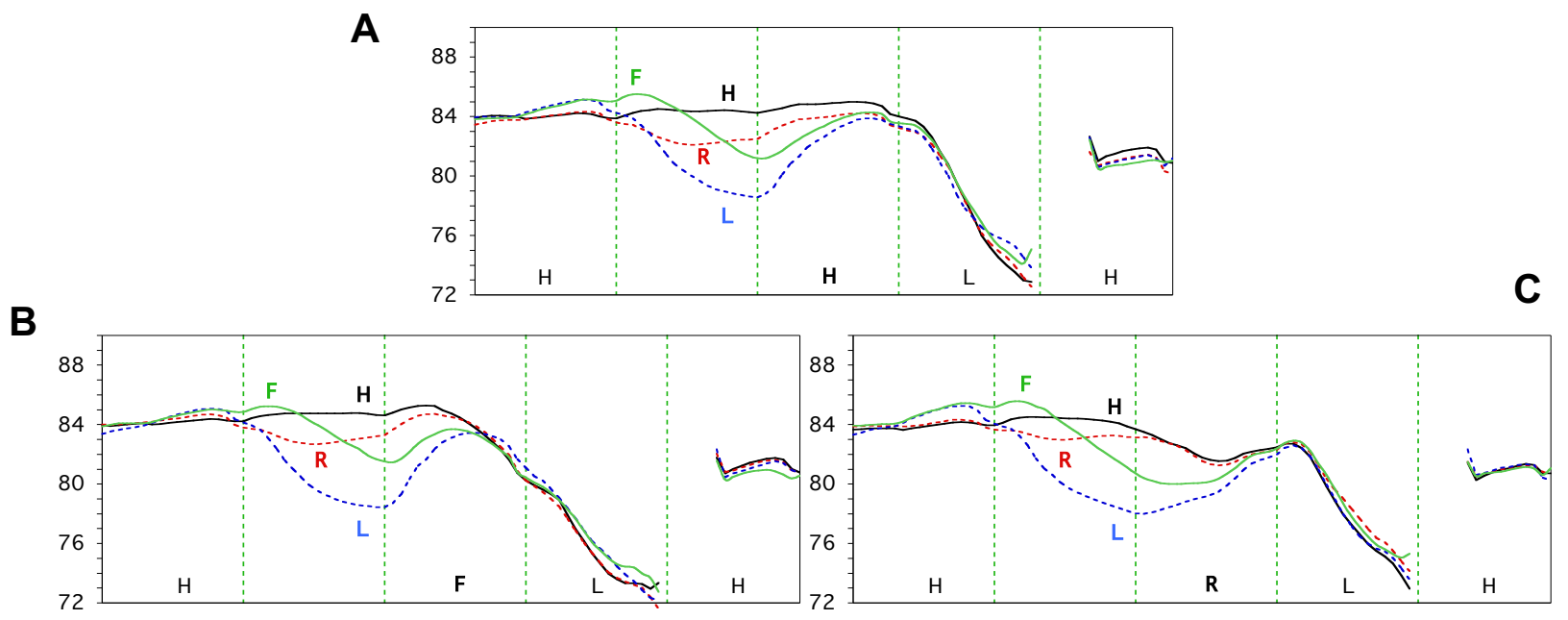

Figure 5. Mean time-normalized $\mathrm{F}_{0}$ contours of Mandarin tones in 5-syllable sentences, where all syllables are in the form of nasal+vowel. In each plot, the tones of all the syllables remain constant except those of the $2^{\text {nd }}$ syllable, which alternate from High (H) to Rising (R), Low (L) and Falling (F). Data from Xu (1999).

\subsubsection{Sequentiality}

In Figure 4, although the surface trajectory is smooth and continuous, the underlying targets are strictly sequential, with neither gap nor overlap around the boundary. Thus there is no need for specifications (hence no extra degrees of freedom) on how much adjacent targets overlap with each other, or whether a temporal interval is targetless. But there are also alternative conceptualizations on the sequencing of targets. One is gestural overlap and the other is intermittent target specifications. Gestural overlap is seen in articulatory phonology (Browman \& Goldstein, 1992a), which assumes that gestures can temporally overlap with each other even 
for the same articulator. Gestural overlap is used to explain anticipatory coarticulation as well as undershoot (Browman \& Goldstein, 1992a, 1992b). The execution of gestural overlap is implemented in the task dynamic model as weighted averages of the overlapped gestures (Saltzman \& Munhall, 1989). There has been evidence, however, that the movements of any single articulatory dimension result from sequential rather overlapping execution of successive targets. This is shown for tongue body (Wood, 1996), velum and lips (Bell-Berti \& Krakow, 1991; Boyce, Krakow \& Bell-Berti, 1991), and $\mathrm{F}_{0}$ (Chen \& Xu, 2006). Also Ostry, Gribble and Gracco (1996) have demonstrated that, a model based on the equilibrium point (EP) hypothesis of motor control (Laboissiere, Ostry \& Feldman, 1996) is able to generate kinematic movements that show coarticulatory overlap with non-overlapping underlying control signals. These findings, however, have so far not been able to offset the appeal of gestural overlap, which remains a widely accepted model of coarticulation (Kingston, 2019; Stevens \& Hanson, 2012). An important reason is the non-unique relations between the observed articulatory/acoustic trajectories and possible underlying control parameters, as illustrated in Figure 6.

In panel (A) of Figure 6 there are three successive target approximation movements, each largely attaining its target by the offset. These movements are strictly sequential, as indicated by the alteration of the line patterns. Panel (B) also shows three target approximation movements, but the first one is shortened relative to the first movement in panel (A), resulting in undershoot, i.e., an incomplete attainment of the target. From the graph it is clear that the undershoot is due to a premature termination of the first movement by the early onset of the second one, which truncates the former. But the truncation also makes the offset of the first movement appear "assimilated" to the second target, as indicated by the arrow. When the time reference (vertical line) remains unchanged from panel (A), the first movement also appears to "anticipate" the second one, although there is no true anticipation given the clearly marked movement boundary. In panel (C), instead of truncation, the final portion of movement 1 and the initial portion of movement 2 are overlapped. The overlap is implemented by inserting a new target that is the average of the first and second targets. ${ }^{3}$ This blending thus explicitly models an "anticipatory assimilation." The resulting trajectory, however, is not very different from the one in panel (B) if the boundaries are ignored ${ }^{4}$. Thus truncation can generate trajectories very similar to those generated by blending, but it has the advantage of not needing to specify the amount of overlap, thus eliminating a critical degree of freedom.

\footnotetext{
${ }^{3}$ There are also other, more sophisticated ways of blending, e.g., averaging, suppressing and adding (Saltzman \& Munhall, 1989).

${ }^{4}$ Compared to panels $\mathbf{A}$ and $\mathbf{B}$, the second target in panel $\mathbf{C}$ is less fully attained. This is because the blending also shortens the target approximation movement of the second target. Thus there is less than enough time to reach the target even though the onset of the movement is actually higher than in the other two panels. This means that despite the similarity, different assumptions about sequential arrangements do lead to slight variations in surface trajectory, making direct computational comparisons possible in future research.
} 
A

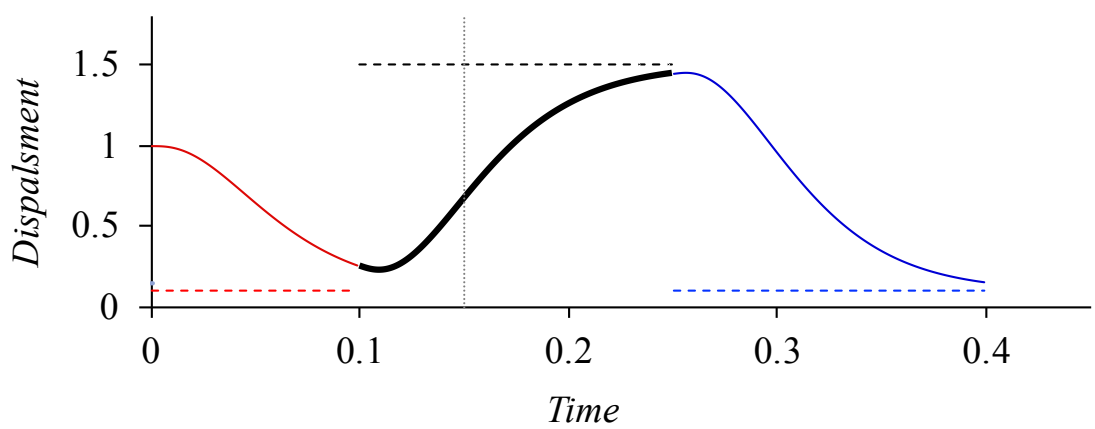

B

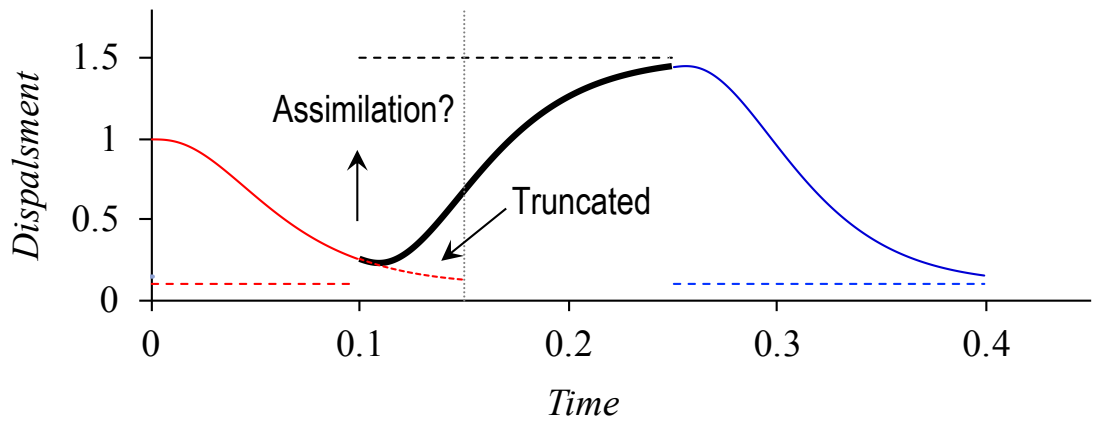

C

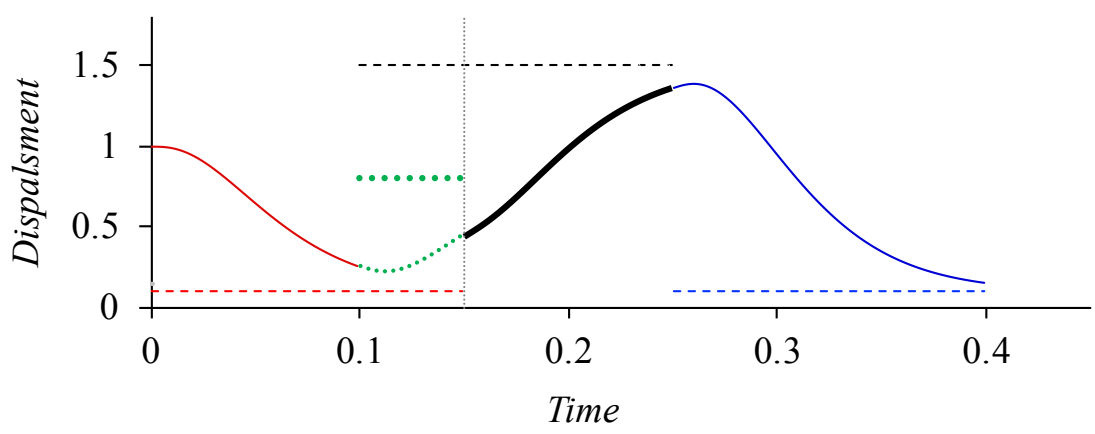

Figure 6. Sequential and overlapping target approximation processes generated with the qTA model (Promon et al., 2009). The units of both axes are arbitrary. In (A), the three target approximation movements are strictly sequential, and the vertical line is the boundary between the first two movements. In (B), the vertical reference remains at time 0.15 , but the first movement is shortened by 0.05 unit. All the movements remain sequential (which means that the "tail" of the first movement- the dotted continuation - is truncated). In (C), the first and second movements overlap with each other by 0.05 units. The overlap is implemented by inserting a blended target (dotted green horizontal line), which is the average of the first two targets.

Sequentiality through truncation has a number of further implications. The first is that duration of target approximation is mostly an extrinsic rather than intrinsic property of the gesture (Fowler, 1980), which allows it to be specified by external functions like word stress, lexical quantity, focus, boundary marking, etc. (Xu, 2009). Secondly, given the frequent occurrence of truncation due to the extrinsic factors (Xu \& Prom-on, 2019) and the fact that any degree of truncation is possible (even up to $100 \%$, e.g., in case of syllable contraction: Cheng \& Xu, $2013)$, target approximation is rarely a $0-360^{\circ}$ full cycle. Thus it is inappropriate to model intergestural alignment in terms of phase relations such as being in-phase or anti-phase (Nam et al., 2009). This would be a further reason, in addition to those listed in Table 1, against modeling 
motor synchrony as physical entrainment. Finally, the massive range of possible truncation (Cheng \& Xu, 2013; Xu \& Prom-on, 2019) would make it impossible to control duration through articulatory strength (Byrd et al., 2000; Edwards, Beckman \& Fletcher, 1991), because, for example, it is inconceivable that an extreme shortening of a segment or syllable up to full elimination is achieved by a maximum increase of stiffness.

\subsubsection{Full vs. underspecified targets}

Underspecification has been a popular idea in both phonology and phonetics to account for severe undershoot or lack of apparent targets (Arvaniti \& Ladd, 2015; Keating, 1988; Myers, 1998; Steriade, 1995). The hypothesis is that some units do not have fully specified phonetic values, and their surface patterns come from interpolation between adjacent, fully specified units. Boyce et al. (1991) have shown, however, that intervals with highly variant lip rounding and nasalization properties may still stem from specific underlying goals, as observed with minimal contrast comparisons of articulatory movements. Similar findings have been made for the neutral tone in Mandarin (Chen \& Xu, 2006; Liu et al., 2013) which has often been considered targetless. In Figure 7(A), the $\mathrm{F}_{0}$ contours of the Falling $(\mathrm{F})$ tone in the second syllable converge quickly to a falling slope following the four full tones in the first syllable. In contrast, the neutral-tone sequence does not show full convergence of $F_{0}$ by the end of the second syllable. But by the end of the third neutral tone in Figure 7(B), all the trajectories have approached a mid-level $\mathrm{F}_{0}$. This approximation indicates that the neutral tone has its own target, which is halfway between the Falling tone and the Low tone, as evident from Figure 7(C). But the slow approximation in Figure 7(B), as opposed to the quick convergence in Figure 7(A), suggests a weak articulatory strength in the realization of the neutral-tone target. Note that the assumption of no underspecification further reduces DOF by eliminating the need to specify for every temporal interval whether some target properties are missing (Fujisaki et al., 2005; Saltzman \& Munhall, 1989).

A

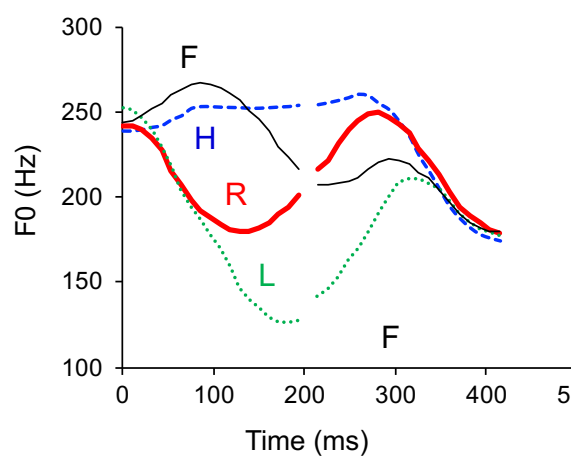

B

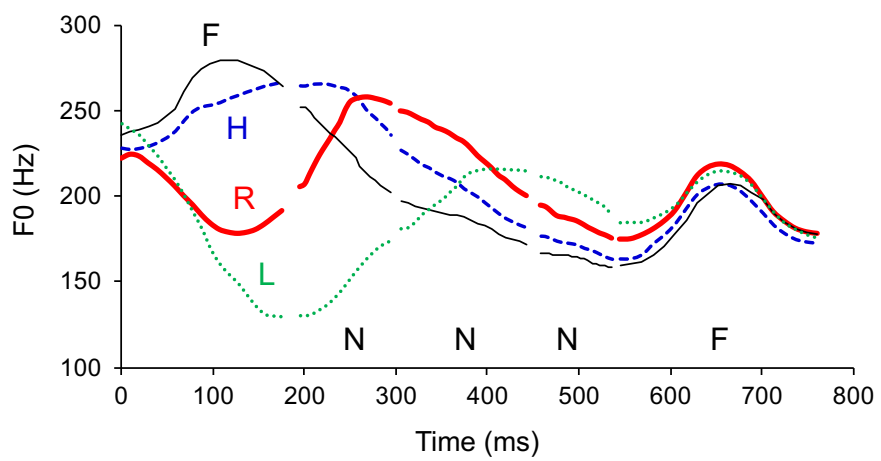




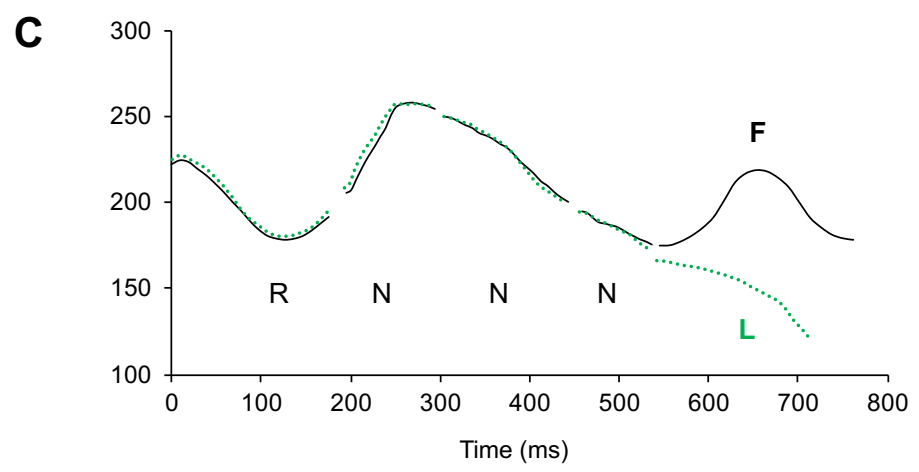

Figure 7. $F_{0}$ contours of the neutral tone versus full tones in Mandarin. (A) Four full tones followed by a Falling tone. (B) The same four tones followed by a sequence of neutral tones. (C) A sequence of neutral tones followed by either a Falling tone or a Low tone. Data from Chen and Xu (2006).

\subsubsection{Target approximation vs. its preparation}

From Figure 7(C) it is also apparent that there is no anticipatory effect of the $\mathrm{F}_{0}$ differences due to tone of the final syllable upon the preceding neutral tones, just as shown in Figure 6. This suggests that there is no need to assume leftward overlap of the full tone target with the preceding target even if it is weak. But anticipatory preparation has been a popular idea for segmental articulation (Kühnert \& Nolan, 1999; Whalen, 1990). Yet the definition of preparation has been unclear. As an illustration, Figure 8 shows the decomposition of a badminton smash, a complex skilled motor action. The goal of the action is to strike the shuttlecock as hard as possible, which is achieved by a unidirectional arcing movement of the racket (frames 4-6). But before that, the racket is moving in the opposite direction in preparation for the main arcing movement (frames 3-4). The function of this preparatory movement is to maximize the travel distance for the racket during the smash, with the goal to achieve a high velocity. Similar preparatory movements have been shown for both singing and speech. For singing, a preparatory movement in the opposite direction from the target note is found to be a core property of the singing voice (Saitou, Unoki \& Akagi, 2005). For speech, pre-low raising, which increases the pitch of a non-low tone before a low-pitched tone, has been reported for a number of languages (Gandour, Potisuk \& Dechongkit, 1994; Gu \& Lee, 2007; Laniran \& Clements, 2003; Lee et al., 2017; Xu, 1997, 1999).

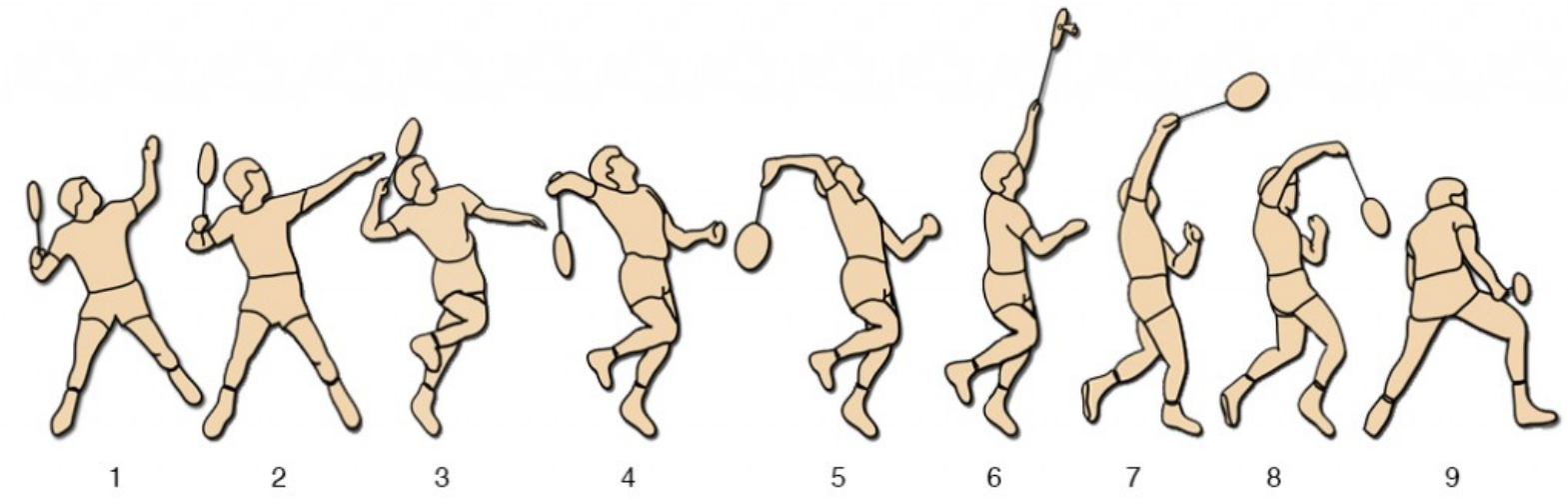


Figure 8. Decomposition of a badminton smash consisting of three phases. Frames 1-4 are the preparation phase, while frames 4-6 are the unidirectional approximation phase. The goal is not only to reach the position of the racket-shuttle contact, but also to achieve a high velocity at the point of contact. Frames 79 are the settling phase (hubpages.com, 2014, courtesy of Michael Hayes at HowTheyPlay.com).

In contrast to the preparatory movements in frames 3-4, the movements in frames 4-6 are all in the direction of making the ultimate contact, and so no part of it, including the initial portion, say frames 4-5, should be taken as preparatory activities separate from the rest of the arcing movement in the same direction. By the same token, the entire unidirectional movement toward a phonetic target should be considered a single target approximation action. This distinction between preparation and target approximation will be highly relevant in the upcoming discussion of coarticulation. ${ }^{5}$

\subsubsection{Dynamic targets and velocity propagation/continuity}

From Figure 5(B, C), we can see that the $\mathrm{F}_{0}$ contours of the Falling tone after four different tones all converge to a linear falling slope, and those of the Rising tone all converge to a linear rising slope. It has also been shown that in both dynamic tones of Mandarin $(\mathrm{Xu}, 2001)$ and Cantonese (Wong, 2006), and diphthongs in American English (Gay, 1968), the final velocity of $\mathrm{F}_{0}$ and formants remains largely constant when speech rate varies from normal to slow. Thus a specific velocity is aimed at as part of the phonetic target associated with those linguistic units. Dynamic targets are actually commonplace in other motor movements. Again from Figure 8, we can see that when the target is reached in frame 6 , what is achieved is not only a particular position of the racket, but also a high-speed impact on the shuttlecock. Thus the target of the smash is dynamic, consisting of both position and velocity specifications. Also, given a high velocity as part of the goal of a dynamic target, its achievement may have a powerful carryover effect on the following movement. In Figure 7(C), for example, the final velocity of the Rising tone is so high that the $\mathrm{F}_{0}$ rise continues for more half of the syllable in the following neutral tone.

\subsubsection{Summary of target approximation}

There is much evidence that continuous surface movements of both articulatory and acoustic dimensions result from strictly sequential approximation of successive targets, and each approximation is executed with a specific articulatory strength. On the other hand, there are also alternative models that assume temporal overlap of tauto-articulator gestures and underspecified targets, and targetless intervals. Although both types of models can theoretically generate contextually variant surface trajectories, strictly sequential and fully specified targets have the advantage of assuming fewer degrees of freedom and offering a simpler basis for defining the onset and offset of articulatory gestures, which is critical for edge synchronization.

\footnotetext{
${ }^{5}$ It is possible, however, that even the preparatory movements in frames 3-4 of Figure 8 is part of the smash action. Whether this is the case could be determined by the timing of the movement sections. In Figure 5, e.g., the anticipatory raising movement, hence the "preparation", before the L and F tones seem to start from the middle of the preceding syllable. That is where the second syllable actually starts, as argued in the subsequent discussion. This possibility will need to be investigated in future research.
} 


\subsection{Edge synchronization ${ }^{6}$}

As shown in Figure 2, edge synchronization means that a) the onset of the syllable is the start of the target approximation for most of the syllabic components, including the initial consonant, the first vowel, the lexical tone and the phonation register ${ }^{7}$, and $b$ ) the offset of the syllable is the end of all the remaining movements. The mechanism therefore entails full synchrony at both edges of the syllable. The synchrony is asymmetrical across the syllable, however. At the left edge, there is synchronous onset of all the syllabic components involved, while at the right edge, there is synchronous offset of only supralaryngeal components with that of either a $\mathrm{C}$ or $\mathrm{V}$, but not both. The benefit of synchronization is already discussed in Section 2, and the following discussion is only on the evidence and manner of realization of edge synchronization.

\subsubsection{C-V synchronization and coarticulation}

As mentioned in 2.4, a major objection to the notion of articulatory syllable (Kozhevnikov \& Chistovich, 1965) is that the scope of the vowel should extend well before the syllable onset based on the classic finding of Öhman (1966). That is, in a $\mathrm{V}_{1} \mathrm{CV}_{2}$ sequence, activity of $\mathrm{V}_{2}$ can be seen during $\mathrm{V}_{1}$, as shown in Figure 9, where F2 starts to rise well before the closure of /b/. Öhman's (1966:165) interpretation this "anticipatory" activity is that "a motion toward the final vowel $\left[\mathrm{V}_{2}\right]$ starts not much later than, or perhaps even simultaneously with, the onset of the stop-consonant gesture." But acoustically, the start of that activity, namely, the rise of F2, is well inside $\mathrm{V}_{1}$, which has given the impression that $\mathrm{V}_{1}$ is coarticulated with $\mathrm{V}_{2}$ (Kühnert \& Nolan, 1999). Here lies, therefore, the key challenge of coarticulation: the discrepancy between articulatory and acoustic onset of a phonetic unit, namely, articulation starts well ahead of acoustics. Now, the explicit definition of sequential target approximation in 3.1 would suggest that, in fact, there is no discrepancy between articulation and acoustics. This is because any acoustic movement away from the target of a sound is by definition no longer part of that sound. By the time F2 starts to turn upward in Figure 9, the articulation of $\mathrm{V}_{1}(/ \mathrm{a} /)$ is already over, and the articulation of $\mathrm{V}_{2}(/ \mathrm{y} /)$ has already started, as illustrated in Figure 6(B). There is therefore no evidence of anticipatory coarticulation of $V_{2}$ with $V_{1}$ in Öhman (1966).

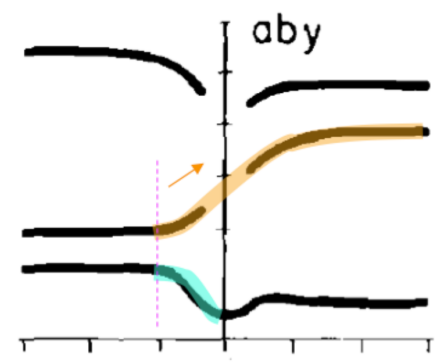

Figure 9. A reinterpretation of Öhman (1966). Reproduced with permission from Acoustical Society of America, with illustrative modifications.

\footnotetext{
${ }^{6}$ The full synchronization of consonant, vowel and laryngeal components at syllable edges was first proposed in $\mathrm{Xu}$ and Liu (2006). But it was not linked to the need to reduce degrees of freedom.

${ }^{7}$ Here phonation refers to the use of voice quality as an independent dimension to mark lexical contrasts in some languages (Huffman, 1987; Wayland and Jongman, 2003). It does not refer to phonation properties that accompany consonant manner of articulation.
} 
But true coarticulation in the sense of co-production (Fowler, 1981) does happen, between syllable-initial consonant and the first vowel of the syllable. In Figure 9, roughly at the time when F2 makes an upward turn, F1 starts to go down toward the low valley in the /b/ closure, indicating that the articulation of $/ \mathrm{b} /$ also starts from there. What is not clear is whether the target approximations of $\mathrm{V}_{1}$ and $\mathrm{C}$ happen exactly at the same time, as Öhman (1966) did not directly compare the timing of the articulation of $\mathrm{C}$ and $\mathrm{V}$. Clearer evidence is seen in $\mathrm{Xu}$ and Gao (2018) based on Mandarin data, which uses formant divergent points of minimal pairs as an indicator of gestural onset. The stimuli were minimal triplets of syllable sequences in the form of $C_{1} V_{1} \# C_{2} V_{2}$, as shown in Figure 10. Each triplet consists of two minimal pairs. The first contrasts the two consonants in $\mathrm{C}_{2}$ : [j] vs. [1], while the second contrasts the two vowels in $\mathrm{V}_{2}$ : [i] vs [u]. In the first minimal pair, the divergent point of the F2 trajectories indicates the onset of $\mathrm{C}_{2}$, because that is where the two consonants start the approximation of their respective places of articulation. In the second minimal pair, the divergent point of F2 indicates the onset of $V_{2}$, because it is where the two vowels start the approximation of their respective vocal tract shapes. The two consonants are both sonorants with incomplete closure of the oral cavity, so that formant movements during the consonantal constrictions can be traced. In addition, all the words have the Rising tone on both syllables, which allow the two resulting $\mathrm{F}_{0}$ peaks to serve as time references for the onset and offset of the second syllable (Xu \& Liu, 2007).

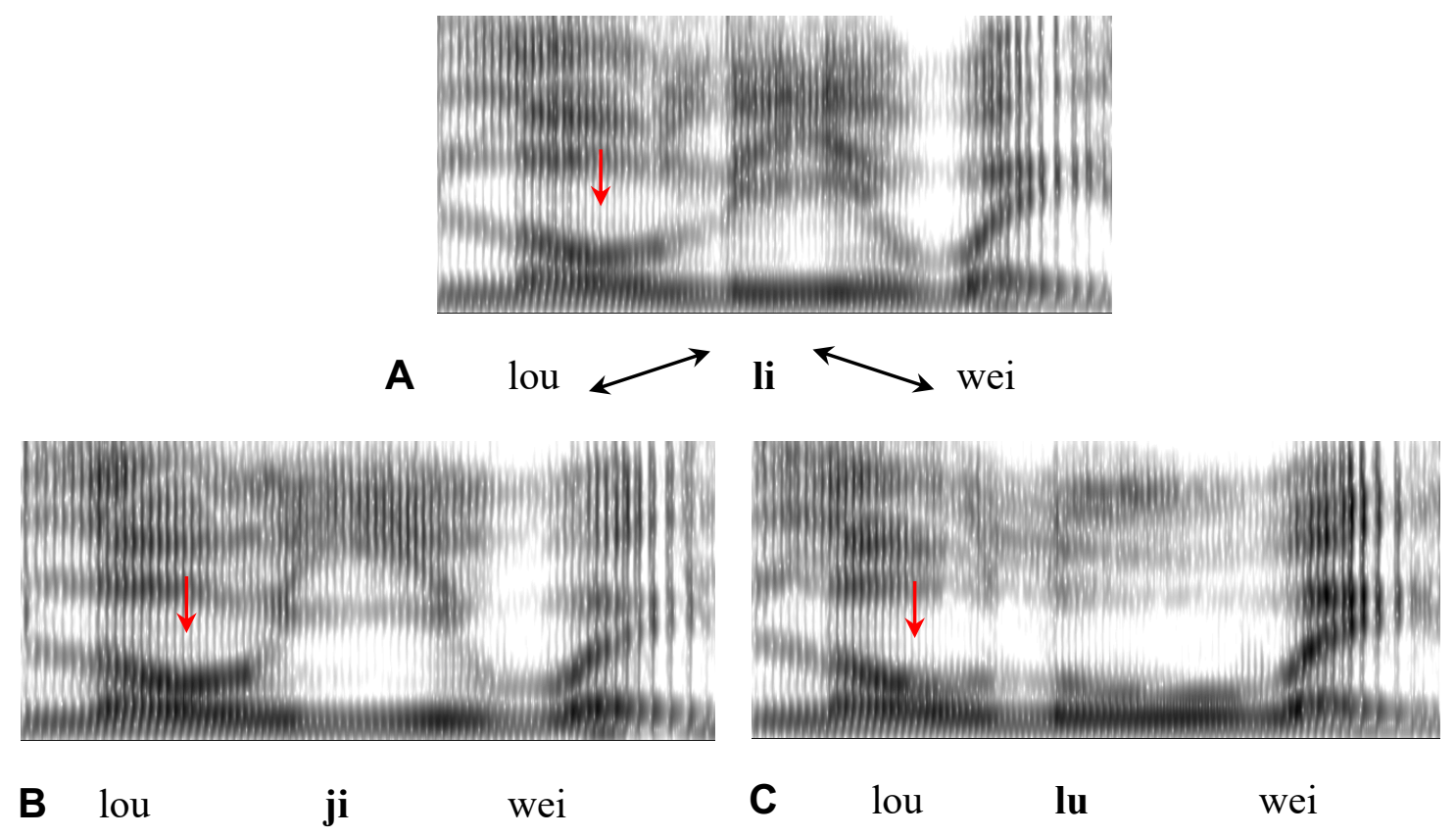

Figure 10. Example spectrograms of a triplet from Xu and Gao (2018). See text for details.

Figure 11 shows mean F2 trajectories of four of the triplets in Xu \& Gao (2018) produced by three male speakers (with 8 repetitions each). In each plot, the solid and dashed lines differ in the initial consonants: [1] vs. [j], and the point at which the two trajectories start to diverge indicates the onset of both consonants. The solid and dotted lines, on the other hand, differ in the vowels of the second syllable: [i] vs. [u], and the point at which the two trajectories start to diverge indicates the onset of both vowels. Strikingly, in each case the vowel divergent point 
occurs at about the same time as the consonant divergent point. This common divergent point, as indicated by the vertical arrows in Figure 10, is well ahead of the onset of the [1] closure (about 50-100 ms based on a preliminary estimate). These data therefore offer, so far, the most unequivocal evidence that consonant and vowel target approximations start at the same time at the onset of the syllable.
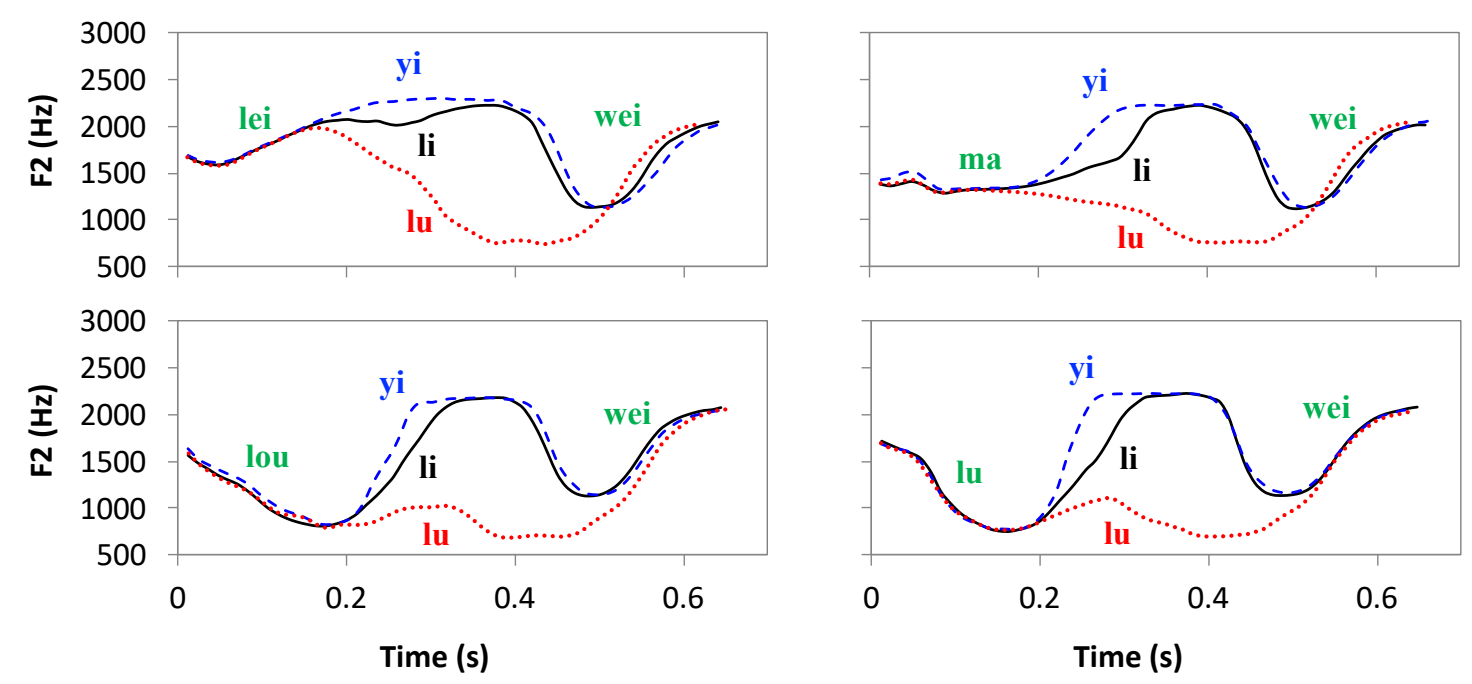

Figure 11. Mean F2 trajectories of the middle three syllables in the Mandarin sentence frame "bi _ wei shan" [more hypocritical than _ _ , where the key word is a disyllabic personal name. The three trajectories in each plot differ either in the initial consonant or the vowel, and the four plots differ only in the first syllable. The time of all trajectories is relative to the voice onset of /bi/ in the carrier. Both F2 and time are averaged across 8 repetitions by 3 male speakers. (Data from Xu \& Gao, 2018)

\subsubsection{Coarticulation resistance}

The coproduction of $\mathrm{C}$ and $\mathrm{V}$ at the syllable onset means that they would interfere with each other's articulation, because they often have conflicting goals. This would result in variations in their acoustic output. Given coproduction as the mechanism of coarticulation, however, there has to be a solution to the articulatory conflict between the coproduced phonetic components. It is known that some segments show better ability to resist coarticulatory variation (Bladon \& Al-Bamerni, 1976; Recasens, 1984a, 1984b). A major source of such coarticulation resistance is the amount of constraint that a consonant or vowel places on the tongue body (Recasens, 1984a, b). Those with intrinsically stronger tongue body constraints show greater resistance to coarticulatory influence than those with weaker constraints.

What can be first recognized is that the severity of the conflict would depend on the number of articulators shared by the co-produced sounds. The least conflict would occur between well separated articulators, e.g., the larynx and the oral articulators, as will be discussed in 3.2.4. The most severe conflict would occur when virtually all the articulators receive clashing demands. This would happen between glides like $/ \mathrm{i} /$ and $/ \mathrm{w} /$ and the following vowel. As semivowels, their articulatory targets specify the shape of the entire vocal tract, just like a vowel. The glide and vowel targets therefore have to be sequentially approximated, as can be seen in Figure 10(B) for $/ \mathrm{w} /$ between /i/ and /ei/. If some of the articulators are shared while others are free to serve either of the two sounds involved, an obvious solution is for the shared 
articulators to sequentially approach different targets, while allowing the rest of the articulators to simultaneously approach their respective targets. In /ba/, for example, the shared articulator, the lips, can first make the bilabial closure and then open up for the vowel. At the same time, all the lingual articulators, with no positional requirement from $/ \mathrm{b} /$, would be free to start approaching their/a/-specific targets from the syllable onset.

The trickiest situation is when two segments share the same primary articulator, as in the case of velar consonants like/ga/, /gi/ and /gu/. Because the tongue body needs to contact the velum, there is a direct conflict with the coproduced vowels, which also have clear specifications for the tongue body. How is it possible, then, for sequential target approximation to resolve the articulatory conflict? Our hypothesis, as already mentioned in 3.1.2, is that sequential target approximation can be specific to individual dimensions of an articulator rather than always over the whole articulator. For the velar consonants, the tongue dorsum needs to be raised to make the velar contact, the vertical position of the tongue dorsum therefore has to first approach the consonant target before turning to the vowel target. The precise horizontal position of the tongue body, in contrast, is probably less critical. Therefore, the horizontal position of the tongue dorsum can start to move toward the vowel target right from the syllable onset. As a result, the point of contact between tongue body and the palate for $/ \mathrm{k} /$ would naturally vary gradiently with the coproduced vowel: more advanced for front vowels, and more retracted for back vowels (Dembowski, Lindstrom \& Westbury, 1998). Conceptually, therefore, dimensionspecific sequential target approximation not only resolves the problem of coarticulation resistance, but also explains how $\mathrm{CV}$ co-onset is articulatorily implemented in general.

A recent modelling study has put the idea of dimension-specific sequential target approximation to test (Xu et al., 2019). An articulatory synthesizer (Birkholz \& Jackel, 2003) in which the dynamics of all articulators are controlled by a target approximation model, was trained with acoustic signals of CVC syllables to learn articulatory targets of consonants and vowels. During learning, the training algorithm allowed tongue dorsum height to be controlled by the velar stop up until the moment of tightest closure, and the tongue dorsum frontness was controlled by the vowel from syllable onset to the vowel offset. Not only was the variable velum contact location successfully learned, but also the $\mathrm{gV}$ syllables synthesized with articulatory parameters learned this way were highly intelligible, with mean recognition rates of $78 \%, 100 \%$ and $83 \%$ for get, god and good, respectively.

\subsubsection{Locus and locus equations}

Coarticulation resistance is also closely related to two other classical phenomena, namely, locus and locus equations. Locus is a phenomenon observed soon after the classical discovery that F2 transitions carry perceptual cues for consonant place of articulation (Cooper et al., 1952). It was found that many of the transitions point back in time to a locus such that as long as the first part of the transition is silent, the same consonant is heard (Delattre et al., 1955; Liberman et al., 1967), as shown in (B) in Figure 12a for /d/. The presence of the entire transition would, in contrast, result in hearing different consonants depending on the following vowel ((A) in Figure 12a). As reasoned by Delattre et al. (1955:772), the locus phenomenon indicates that "no appreciable sound is produced until at least part of the articulatory movement has been completed." But it is left unexplained why the early part of the transition movement has to be silent. 
a.

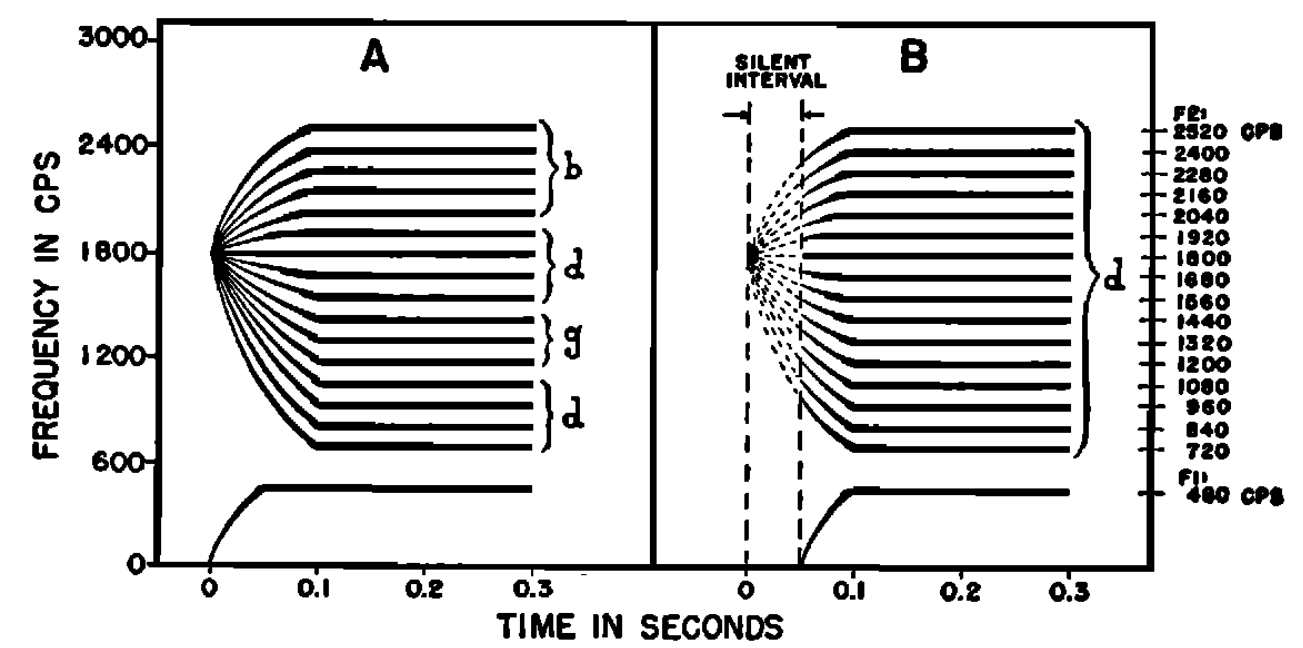

b.

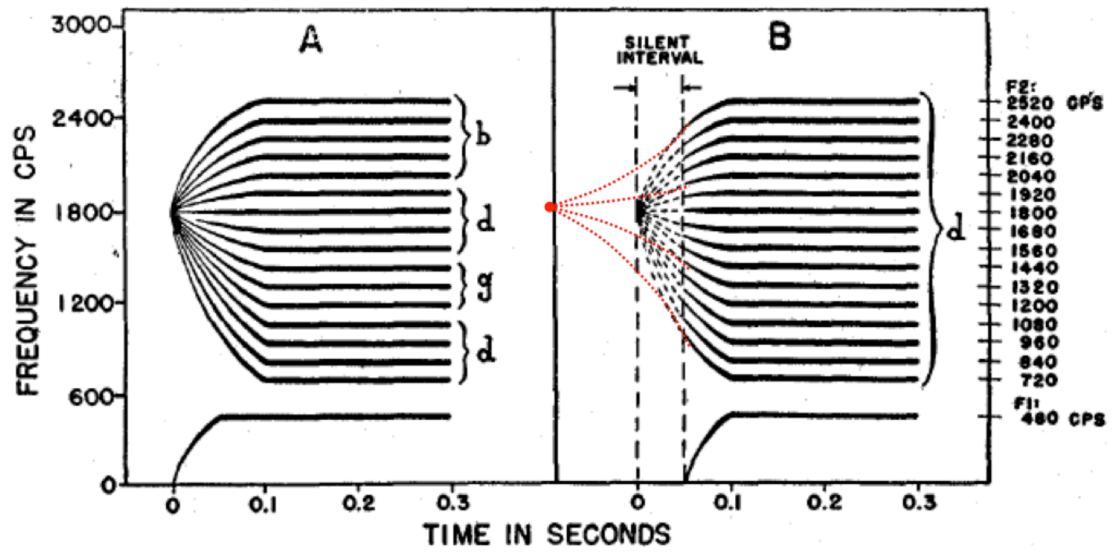

Figure 12. a. An illustration of the locus phenomenon (Delattre et al., 1955. Reproduced with permission from Acoustical Society of America). The curves are F1 (the curves near the bottom in both plots), and F2 hand-painted for the pattern playback speech synthesizer (Cooper, Liberman \& Borst, 1951). b, d and g mark how listeners identified the consonants. $\boldsymbol{b}$. A reinterpretation of Delattre et al. (1955).

Closely related to the locus phenomenon is locus equations (Lindblom, 1963; Lindblom \& Sussman, 2012). Locus equations refer to the finding that the onset of vowel F2 transition after a given stop consonant (i.e., the equivalent of the second vertical dash in Figure 12b(B)), when plotted over F2 at the "centre" of the vowel (equivalent of the plateau in Figure 12b(B)) across different vowels, shows a strong linear relation. Fowler (1994) and Iskarous et al. (2010) have linked the linearity in the locus equations to coarticulation resistance, arguing that it demonstrates the invariance in coarticulation resistance across different vowels. Lindblom and Sussman (2012) have further linked locus equation back to the classical locus phenomenon, proposing that the critical articulation of a stop consonant is the target: lips for $/ \mathrm{b} /$, tongue blade for $/ \mathrm{d} /$, and tongue body for $/ \mathrm{g} /$, but the rest of the articulators have no specified target and so are allowed to be coarticulated with the vowel. This account comes very close to the 
coarticulation resistance account by Fowler and colleagues. Common to both accounts, however, is that they are concerned only with the articulatory or formant movement from the voice onset to the centre of the vowel. Lindblom and Sussman (2012:17) explicitly assumed that "the movements of the articulators in a CV syllable start from their positions at stop closure."

Based on the discussion of edge synchronization thus far, the movement toward the vowel in a CV syllable starts neither from the voice onset after the consonant release, nor from inside the closure, but from the onset of the final formant transition in the preceding syllable, as illustrated by the dotted curves in (B) of Figure 12b. Even if the syllable is utterance initial, as in the case of Delattre et al. (1955), vowel target approximation also would have begun before the consonant closure, i.e., at the time when the consonant closure starts to form. Hence, the onset of the visible formant transition is well after the onset of vowel articulation. This perspective has two implications. Firstly, the linearity of the locus equations is largely due to a part-whole correlation (Benoit, 1986; Löfqvist, 1991; Munhall, 1985), since the two F2 measurements are taken from two locations along the same unidirectional movement from the consonant to the vowel: voice onset which is virtually a halfway point of the $\mathrm{C}-\mathrm{V}$ transition, and center of the vowel, which is the end of the vowel target approximation. Secondly, because the vowel is coproduced with the consonant at syllable onset, and coarticulation resistance is the result of coproduction (depicted in (B) in Figure 12b as the warping of the dotted curves) whose severity depends on how much the consonant articulation conflicts with that of the vowel, the slope of the locus equations would naturally reflect the amount of coarticulation resistance.

\subsubsection{Synchronization of laryngeal and supralaryngeal articulations}

As early as 1984, Ohala and Kawasaki have suggested that "the division of sound sequences into syllables" is "for the sake of synchronizing the segmental and suprasegmental articulations" (p. 123). This proposal foreshadowed a series of later findings in both tone and non-tone languages that generated five lines of evidence for the full synchrony of tone and syllables. The first line of evidence is that the start of tonal movement is aligned to syllable onset. The clearest cases are from tone languages, for which it is possible to directly observe where different tones start to move toward their respective targets (Xu, 1997, 1998, 1999, 2001). In Figure 5, for example, in each plot the $\mathrm{F}_{0}$ contours of the four tones start to diverge roughly from the onset of the second syllable toward their respective targets. Similar consistent start of $\mathrm{F}_{0}$ movement toward tonal targets at syllable onset has also been reported for Cantonese ( $\mathrm{Gu}$ et al., 2007; Wong, 2006) and Shanghai Chinese (Ling \& Liang, 2015). For non-tone languages, there have been many findings of systematic alignment of $\mathrm{F}_{0}$ turning point to syllable onset, e.g., Dutch (Caspers \& van Heuven, 1993; Ladd, Mennen \& Schepman, 2000), Spanish (Prieto \& Torreira, 2007), Greek (Arvaniti et al., 1998), English (Ladd et al., 1999; Xu \& Xu, 2005), Italian (D’Imperio, 2001), Portuguese (Frota, 2002), German (Atterer \& Ladd, 2004), Arabic (Yeou, 2004) \& Persian (Sadat-Tehrani, 2009).

The second line of evidence is that tonal target approximation starts from syllable onset even if the initial consonant is voiceless. As found in $\mathrm{Xu}$ and $\mathrm{Xu}$ (2003) for Mandarin and Wong \& $\mathrm{Xu}$ (2007) for Cantonese, when $\mathrm{F}_{0}$ contours are time-normalized with respect to the syllable, they parallel each other closely whether the onset consonant is a sonorant as in [ma] or a voiceless one as in [ta], [ $\left.\mathrm{t}^{\mathrm{h}} \mathrm{a}\right]$ or [sa]. Thus the approximation of the underlying tonal target starts 
not from the voice onset, but from the beginning of the syllable, regardless of whether the vocal folds are vibrating during the initial consonant.

The third line of evidence is that tonal target approximation ends at syllable offset even if there is a coda consonant. As found in Xu (1998), in disyllabic words with or without a nasal coda in the first syllable, the final part of tonal target approximation is executed through the coda nasal as if it is part of the first syllable. This indicates that the entire syllable is the domain of tonal target approximation whether or not it has a nasal coda, as long as the next syllable does not start with an approximant or a vowel.

The fourth line of evidence is that synchronization of laryngeal and supralaryngeal articulation may benefit vocal learning by eliminating temporal degrees of freedom (Xu \& Prom-on, 2015). As simulated with the qTA model, the learning of tones from raw acoustic contours in real speech data yields both faster and more efficient tone learning when tonal targets are assumed to be fully synchronized with the syllable than when the tone-syllable alignment is assumed to be flexible and has to be learned.

Finally, if $\mathrm{C}$ and $\mathrm{V}$ both start their target approximation earlier than the conventional syllable boundary as has been argued, so should lexical tones. This seems to be indeed the case as can be seen in Figure 5 where the $\mathrm{H}$ tone in syllable 3 is followed by the $\mathrm{L}$ tone. $\mathrm{F}_{0}$ starts to drop toward the low target not at the conventional syllable boundary where the vertical line is drawn, but well before it (but also see discussion in 3.1.4). Further research is needed, however, to systematically examine this particular aspect of tone-syllable alignment.

\subsubsection{Unresolved issues}

There are two issues related to edge synchronization that cannot yet be resolved based on existing data. The first is the temporal alignment of consonant clusters, and the second is vowel harmony. The first issue is about how consonants in a cluster aligned to each other and to the vowel. Browman and Goldstein (1988) proposed a C-center hypothesis, which posits that it is the center of a syllable-initial cluster that is aligned with the vowel. The hypothesis is based on the observation that the most consistent temporal distance from the vowel offset is to the center, rather than the onset or offset of a syllable-initial consonant cluster. A theoretical difficulty with this proposal is the lack of justification for using the vowel offset as the alignment anchor point. Also, further empirical data suggest that the exact temporal relations between consonant gestures with the vowel depends on the syllable affiliation of each of the consonants in a cluster, i.e., whether resyllabification is involved (Byrd, 1995). Browman and Goldstein (2000) further propose that the $\mathrm{C}$-center effect can be accounted for by a model in which all the consonants in a cluster compete to align with the vowel. This is later developed into the coupled oscillation model of gestural alignment (Goldstein et al., 2006; Nam et al., 2009; Saltzman et al., 2008). For one thing, this solution would leave the DOF problem unresolved, as discussed in Section 1. For another, the proposal would make English different from Russian where vowel onset is said to be aligned to the very first consonant in a cluster (Kozhevnikov \& Chistovich, 1965), as mentioned in 4.1. From the perspective of edge synchronization, both scenarios are plausible, as gestural synchrony can be realized only to the degree allowed by sequential target approximation, driven by the same mechanism underlying coarticulation resistance. Finally, the assessment of gestural alignment is highly dependent on how the temporal intervals of gestures 
are defined and measured, as discussed in detail in 3.1. Further studies on the alignment of consonant clusters therefore need to take all these concerns into consideration.

Vowel harmony is the phenomenon that in some languages, within a relatively large temporal domain such as word or phrase, there is a tendency for vowels to share a particular property along a phonetic dimension, such as tongue height, or tongue frontness (Clements, 1976; Nguyen \& Fagyal, 2008). Such long-distant assimilation can be either left to right or right to left. Related to the right-to-left harmony is the long-distance anticipatory vowel to vowel assimilation across more than one syllable reported for English, which is referred to as a form of coarticulation (Magen, 1997; Grosvald, 2010). Both kinds of long-distance assimilation seem incompatible with either target approximation or TD/AP, as it would mean that the approximation of a single vowel target can occur across multiple target approximation movements of the intervening consonants and vowels. The target approximation model, however, allows a distinction between target assignment and target approximation (Xu, 2005). That is, it is possible for the target of a phone, be it segmental or suprasegmental, to be modified, or reassigned, before the start of its articulatory execution. The target reassignment, if extensive enough to be heard as a different vowel, could have originated historically from surface assimilation (Gafos \& Stephan, 2006), due to listeners' misperception (Ohala, 1994; Ohala \& Kawaski, 1984). Or it could be a small target readjustment in anticipation of an upcoming vowel (Nguyen \& Fagyal, 2008) or consonant (Heid \& Hawkins, 2000; West, 1999). The separation of target assignment and target approximation as difference processes would allow the synchronization of the articulation of consonants and vowels, as has been demonstrated in a preliminary study of French (Chiu et al., 2015).

\subsubsection{Summary and impact of edge synchronization}

Multiple lines of evidence have been presented that consonant, vowel and tone are likely synchronized by their onset at the beginning of a syllable. There is also a synchronization tendency at the offset of the syllable, although the evidence is only in terms of tone-syllable alignment. More discussion of syllable offset will be done in light of tactile anchoring in the next section.

A major impact of the edge synchronization hypothesis is an overhaul to the way we conceptually segment speech, as illustrated in Figure 13. Unlike the conventional segmentation in Figure 13(A), in Figure 13(B), the onset of each segment is at a time when the spectrogram starts to move toward its prototypical configuration. For the first /a/, for example, it is in the middle of the conventional /i/ where F2 starts to drop, and for the second /i/, it is in the middle of the conventional /a/ where F2 starts to rise. Thus the onset of a vowel is fully aligned to the onset of the initial consonant, which is also shifted leftward: to where an oral closure just starts to form, as indicated by the downward turn of F1. As explained earlier, the large leftward shift of a vowel onset (by about $100 \mathrm{~ms}$ ) is a conceptual change that can explain away much of the anticipatory $\mathrm{V}$-to- $\mathrm{V}$ coarticulation. The new offset of a segment is where the spectral pattern has maximally approached its canonical configuration and started to move away from it. For /i/, it is at the peak of F2 and F3, for /a/ it is at the peak of F1 and the valley of F2, and for /w/ it is at the valley of F2. For the obstruent consonants, the offset is not at the end of its prototypical spectral pattern (e.g., closure gap in $/ \mathrm{b} /$, nasal or lateral formants in $/ \mathrm{m} / \mathrm{and} / \mathrm{l} /$, and the frication in $/ \mathrm{s} /$ ), but in the middle of these intervals. Furthermore, a coda consonant, e.g., $/ \mathrm{n} /$ 
in /shan/, is aligned after the nuclear vowel, without overlap. The reason for the lack of VC overlap will be discussed in 3.3.2. Finally, tones are fully synchronized with the entire CV or CVC syllable, as shown in the bottom tier.

A

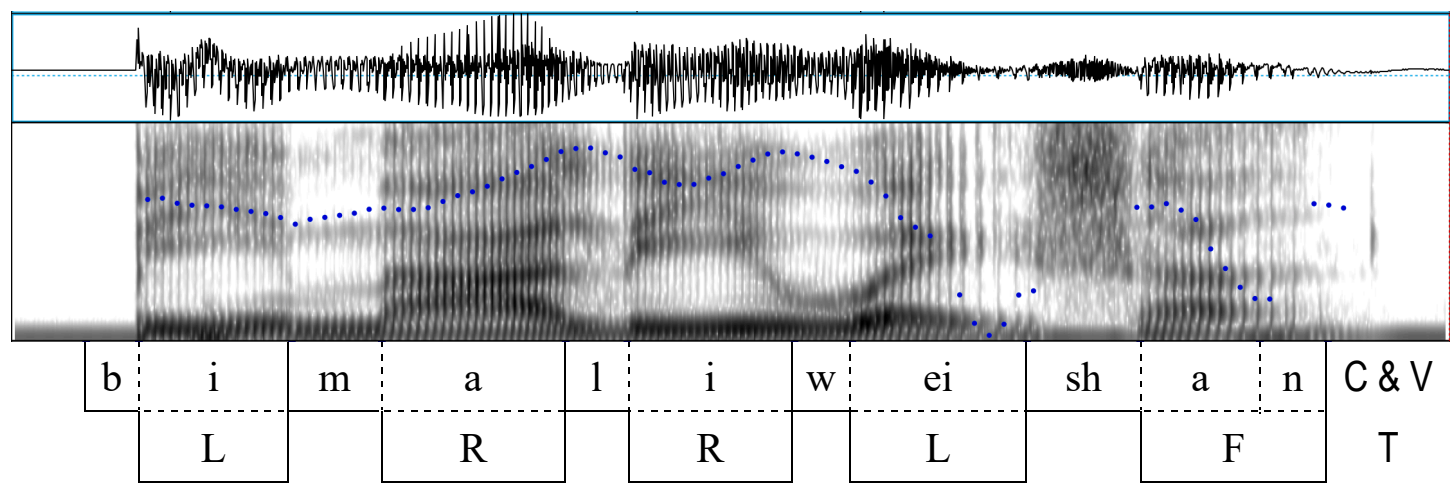

B

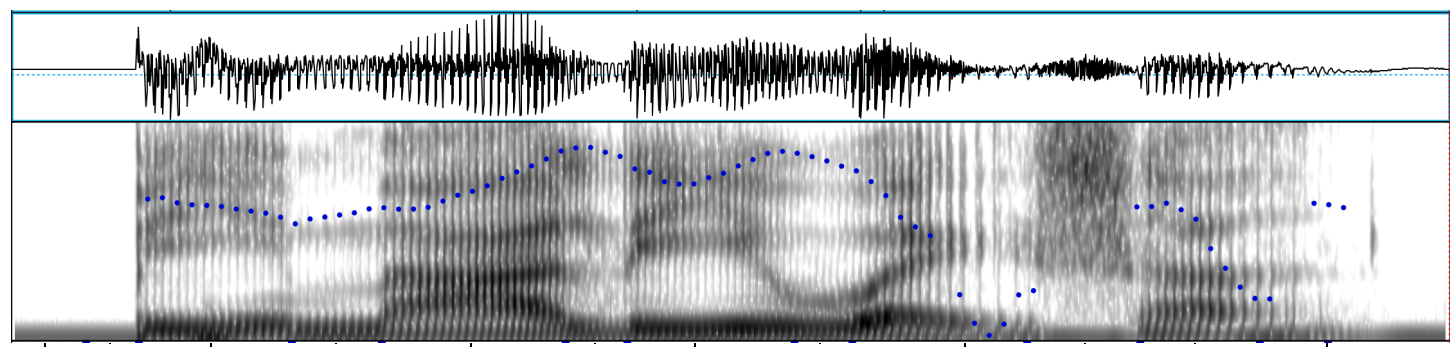

\begin{tabular}{|c|c|c|c|c|c|c}
$\mathrm{b}$ & $\mathrm{m}$ & $\mathrm{l}$ & $\mathrm{w}$ & $\mathrm{sh}$ & $\mathrm{n}$ & $\mathrm{C}$ \\
$\mathrm{i}$ & $\mathrm{a}$ & $\mathrm{i}$ & $\mathrm{ei}$ & $\mathrm{a}$ & & $\mathrm{V}$ \\
\hline $\mathrm{L}$ & $\mathrm{R}$ & $\mathrm{R}$ & $\mathrm{L}$ & \multicolumn{2}{c}{$\mathrm{F}$} & $\mathrm{T}$ \\
\hline
\end{tabular}

C

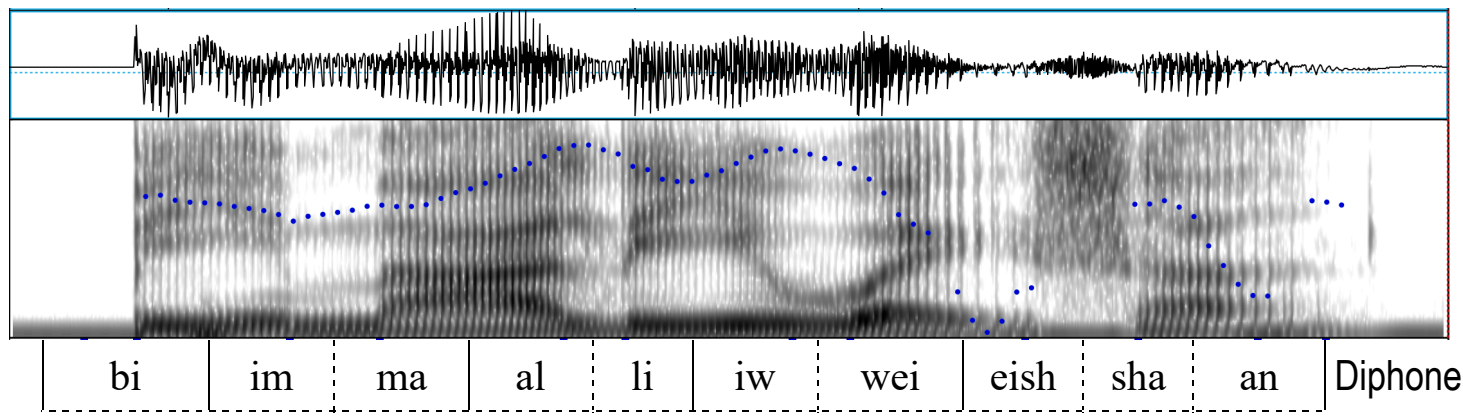

Figure 13. Spectrogram of the Mandarin phrase “比麻黎伪善”/bǐ má lí wěi shàn/ [more hypocritical than $\mathrm{Ma} \mathrm{Li]} \mathrm{(Xu} \mathrm{\&} \mathrm{Gao,} \mathrm{2018),} \mathrm{with} \mathrm{broad} \mathrm{phonetic} \mathrm{transcriptions.} \mathrm{In} \mathrm{both} \mathrm{panels,} \mathrm{C,} \mathrm{V} \mathrm{and} \mathrm{T}$ stands for consonant, vowel and tone, respectively. In (A) the segmentation is conventional (Jakobson et al., 1951; Turk et al., 2006). The segmentation of $/ \mathrm{w} /$ is based on Peterson and Lehiste (1960). In (B) the segmentation is based on the synchronization hypothesis.

Just as importantly, Figure 13(B) makes it clear that the coproduction of consonant and vowel is in fact acoustically transparent rather than hidden. For example, the rise of F2 and F3 toward the high extremes of $/ \mathrm{i} /$ in $/ \mathrm{li} /$ from the middle of the conventional /a/ is clearly visible. Also apparent is the start of the F1 drop toward the low extreme in /1/ from the middle of the 
conventional /a/, which marks the onset of oral closure for both /1/ and /i/. Currently, these visible patterns are given names like transitions, anticipatory movements, etc. The new segmentation treats them, instead, as belonging to the main bodies of the segments.

The representation of the true segmental intervals by the new segmentation shown in Figure 13(B) is in fact foreshadowed by the concept of diphone in speech technology (Taylor, 2009). A diphone is defined as an acoustic chunk consisting of two adjacent halves of a pair of conventionally segmented phones (Peterson, Wang \& Shearme, 1958; Taylor, 2009). As illustrated in Figure 13(C), each diphone extends from the middle of one conventional phone to the middle of the next. Intriguingly, the diphone boundaries in Figure 13(C) match well with those of the new segmental intervals in Figure 13(B). This means that a diphone actually represents a single phone rather than two phones in many cases, especially in the case of consonants. For example, the diphone [im], [al], [iw], [eish] in Figure 13(C) actually represent the full scope of $[\mathrm{m}],[1],[\mathrm{w}]$ and [sh], respectively. But diphone representation of vowels are incomplete compared to the new segmentation scheme, because it misses the initial portion of the vowel. For example, the vowel interval in [ma] in Figure 13(B) spans across two diphones in Figure 13(C): [im] and [ma], and the vowel interval in [li] spans across [al] and [li]. Missing the initial portion of the vowel in the diphone segmentation therefore is likely a major reason why so many contextual features are needed in training a diphone synthesis or recognition system.

\subsection{Tactile anchoring}

Tactile anchoring is about how synchronization is achieved in speech production, and it may hold the key to understanding some of the structural details about the syllable. The hypothesis is that the accuracy of edge synchronization rests on sensory feedback, and that tactile sensations generated during articulation likely provides the most useful feedback information. Given that, the points of synchronization are at the syllable edges rather than in the center of the syllable thanks to tactile anchoring. Most previous theories of the syllable regard the center, where sonority is the highest, as the core of the syllable (see detailed review by Ohala, 1992). Tactile anchoring predicts, in contrast, that the center of the syllable, where contact sensation is likely weak, would be the least reliable anchors.

\subsubsection{Why is tactile anchoring needed?}

One of the earliest clues comes from the finding that, just like bimanual synchronization (Kelso, 1984), concurrent leg swinging by two people sitting next to each other also shows stable phase relations only at $0^{\circ}$ and $180^{\circ}$, and $0^{\circ}$ is the only stable relation at high speed (Schmidt et al., 1990). But this holds only when the participating subjects could see each other's movements. This perceptual nature of motor synchrony is further demonstrated by Mechsner et al. (2001), which shows that the propensity for, as well as the ability to achieve bimanual synchrony are perceptual in nature. They demonstrate that naïve subjects are able to perform bimanual oscillations in a 4:3 frequency ratio, which are virtually impossible to maintain based purely on body-oriented strategies, as long as they can see a 1:1 frequency ratio converted from their actions by a mechanical device. Beside visual perception, tactile (Buchanan \& Ryu, 2005; Johansson \& Flanagan, 2009; Kelso et al., 2001; Koh et al., 2015) and proprioceptive (Baldissera et al., 1991; Mechsner et al., 2007; Ridderikhoff et al., 2007; Spencer et al., 2005; 
Wilson et al., 2003) information has also been shown to help stabilize in-phase coordination in bimanual tasks. Thus the perceptual guidance needed for motor synchrony includes any sensory feedback, and the importance of each perceptual channel is a function of the clarity of the feedback information it provides to the central control system.

For speech, to achieve synchrony in syllable articulation, visual feedback is unlikely to be useful, as speakers cannot see their own articulators. Auditory feedback is available all the time, but it may not be the most critical, as people who become deaf postlingually are often able to speak intelligibly for decades (Cowie \& Douglas-Cowie, 1983; Lane \& Webster, 1991). Also abundant during speech is proprioceptive feedback, but the information it provides is likely spread evenly over time, thus may be useful but not the most critical. The sensory information that probably fluctuates the most with the opening and closing of the vocal tract would be from tactile feedback, and especially from the articulators that are rich in tactile receptors, such as tongue tip, tongue blade and the lips (Lyubimova et al., 1999; Ringel \& Ewanowski, 1965). This would point to consonants as the most likely cites of tactile anchoring, because they, by definition, all involve closures of one kind or another along the vocal tract, which are brief and exact in time.

\subsubsection{Evidence for tactile anchoring in speech}

Tactile anchoring is the most hypothetical component of the new syllable theory, as there is only indirect evidence so far, in terms of its ability to explain various unrelated phenomena. The first is that in consonants that involve multiple articulatory components, the gestural components with a tighter oral contact tend to be aligned closer to syllable edges. In English, for example, the apical gesture in $/ 1 /$ reaches its extreme near the syllable margin, whereas its dorsal component reaches the extreme close to the nuclear vowel, whether $/ 1 /$ is a coda (hence the dark variant) or an onset (hence the light variant) (Sproat \& Fujimura, 1993). This means that gestures that generate clearer tactile feedback are preferred at syllable edges over those that provide less clear tactile information. That is, the apical gesture of /1/ involves a tongue tip contact with the alveolar ridge, hence the rich tactile sensation at the tongue tip would provide much more sensory feedback than the more vowel-like tongue body gesture (Ringel \& Ewanowski, 1965). A similar finding is that in /w/, the labial gesture is also more peripheral than the tongue body gesture (Gick, 2003). Not only does the labial gesture of /w/ involve more skin contact than the tongue body gesture, but also the lips have a rich sensory representation (Ringel \& Ewanowski, 1965).

The second phenomenon is the well-known onset-coda asymmetry, i.e., CV syllables are much more common than VC and CVC syllables, both within and across languages (Clements \& Keyser, 1983; Hooper, 1972, Levelt et al., 1999; Locke, 1983; Ohala \& Kawasaki, 1984; Selkirk, 1982; Vennemann, 1988). Even if they are already present in a language, coda consonants are more vulnerable than onset consonants, as they are subject to reduction, deletion and resyllabification (Dell, 1988; Gao \& Xu, 2010; Schiller, Meyer \& Levelt, 1997; Xu, 1986). The vulnerability of coda means that it is not as reliable as onset for providing tactile feedback. As for why the onset/coda asymmetry is there in the first place, there are a number of reasons. First, syllable onset is where the greatest number of syllabic components can be synchronized, including consonant, vowel and tone, as mentioned before. In contrast, syllable offset can end with either a vowel or a coda consonant, but not both. This is because the closure of a coda 
consonant is in direct conflict with the opening movement of the preceding vowel. This differs from the syllable onset, where it is tolerable for a vowel to be briefly interrupted by the closing movement of the initial consonant. The sequential articulation of codas is likely one of the major reasons for their vulnerability to reduction and deletion them (Xu \& Liu, 2006). As a syllable shortens when speech rate increases, there is less and less time left to allow sequential execution of multiple segments within the syllable (Cheng \& $\mathrm{Xu}, 2013$ ). This vulnerability means that syllable onset is the only temporal location for generating reliable tactile input. Furthermore, because target approximation is frequently uncompleted (Xu \& Prom-on, 2019), and different syllabic components may have different degrees of incomplete approximation, synchronizing their offsets would be hard. Most importantly, syllable offset is also the onset of the next syllable which already provides a synchronization point. So, there is no need for a coda to perform synchronization except at the end of an utterance.

The onset-coda asymmetry is also reflected in the resyllabification phenomenon, whereby a coda consonant has gone through changes that make it sound like the onset of the next syllable. This may happen within a word, e.g., ending, producing, which becomes en-ding, pro-du-cing, or across words, e.g., let us, thin air, which become le-tus, thi-nair. In language teaching, such resyllabification (often referred to as linking) is considered a good marker of fluency for languages like English, as non-native speakers often fail to do it (Anderson-Hsieh et al., 1994; Hieke, 1984). There is doubt, however, as to whether resyllabification actually occurs, especially across word boundaries (Shattuck-Hufnagel, 2011). One of the reasons is that studies of resyllabification relying on either researcher's own intuition or native listener's judgment have generated diverse findings (Chiosáin et al., 2012; Goslin \& Frauenfelder, 2001; Schiller et al., 1997; Treiman \& Danis, 1988).

Gao and $\mathrm{Xu}$ (2010) used a more objective method to determine syllable affiliation of intervocalic nasals at word boundaries in Southern British English. Like in Xu and Liu (2007), $\mathrm{F}_{0}$ contours were used as an independent reference, as shown in Figure 14. As can be seen, the initial nasal murmur occurs mostly to the right of the $\mathrm{F}_{0}$ valley. The nasal geminate seems to be split in the middle by the $\mathrm{F}_{0}$ valley, with the right portion largely equivalent to the initial nasal, while the left portion extending much further back into the first syllable than the initial nasal into the second syllable. Compared to these two cases, the coda nasal is aligned more like the initial nasal, rather than the left portion of the nasal geminate. Compared to both the initial nasal and the right portion of the nasal geminate, however, the duration of the coda nasal is much shorter, a phenomenon Lehiste (1960) found many years ago in cases like a nice man vs. an iceman.

The shortened duration of the coda nasal seems to reflect speaker's knowledge of its underlying morphological association. Thus the coda nasal in these cases could be said to be ambisyllabic, since its $\mathrm{F}_{0}$ alignment is characteristic of initial nasal while its duration still reflects its coda identity. But in terms of how the onset of laryngeal and supralaryngeal articulations are aligned to each other, the coda nasal behaves like an initial nasal. 

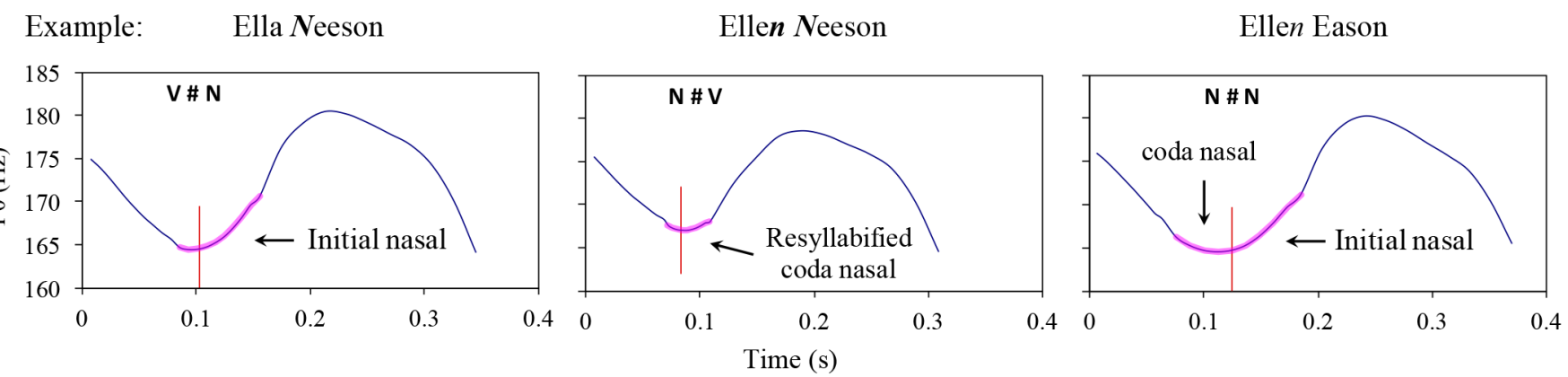

Figure 14. Mean $F_{0}$ contours and location and duration of the intervocalic nasals (thick section of each curve) in mean real time, averaged across 3 sets of 7 sentences by 8 Southern British English speakers, each producing 7 repetitions. The symbol \# indicate word boundary. The words on top of each graph is a representative of the 7 sentences in that set. The italicized letter indicates the target nasal consonant. The short vertical lines show the average locations of the $F_{0}$ valleys. (Data from Gao \& Xu, 2010).

The propensity for resyllabification is further seen in a phenomenon first observed by Stetson (1951). He found that a CVC sequence such as pup, pup, pup..., when spoken at an increasing speech rate, changes abruptly at some point to a CV sequence: $p u, p u, p u \ldots .$. Kelso, Saltzman and Tuller (1986), in a more formal experiment, show that a VC sequence like ip, ip, ip ... also changes abruptly to $p i, p i, p i$... when speaking rate is increased to about 4 syllables/s. A similar finding was made by de Jong (2001). An abrupt shift at 4 syllables/s is striking, since normal speech rate is much faster, at about 5-7 syllables/s (Eriksson, 2012; Tiffany, 1980). This means that resyllabification is virtually inevitable in normal speech, unless codas are deleted when the following syllable starts with a vowel, as is the case in Mandarin (Xu, 1986).

\subsubsection{Summary of tactile anchoring}

The need for tactile anchoring is evident from the finding that the quality of bimanual synchrony of cyclic movements is contingent on the quality of perceptual guidance during the execution of a synchronization task (Mechsner et al., 2001 and many others cited above). Assuming that motor synchrony is the essence of the syllable as currently hypothesized, its accuracy would require clear feedback guidance. Of all the sensory channels available during speech production, the intermittently fluctuating tactile feedback from consonants would provide the most precise feedback. Given the vulnerability of codas, the only temporal location for tactile feedback is syllable onset. This hypothesis is supported by the fact that in sonoront consonants $/ 1 /$ and $/ \mathrm{w} /$, the tongue tip and labial gestures that would generate rich tactile information is realized near the syllable edges, while the tongue body gestures are realized toward the center of the syllable (Gick, 2003; Sproat \& Fujimura, 1993), and by the onset-coda asymmetry that strongly favours $\mathrm{CV}$ over VC or CVC. Further support in terms of the onset-coda asymmetry comes from the strong tendency for resyllabification of coda consonants to the onset of the next syllable (de Jong, 2001; Gao \& Xu, 2010; Kelso et al., 1986; Stetson, 1951).

\section{Neural prerequisites for syllable articulation}

The discussion so far has presented mechanistic reasons why the syllable is likely a synchronization mechanism for reducing degrees of freedom to makes speech possible. No 
neural evidence has been presented, however, because there are no known neural properties, to my knowledge, that would directly implicate a synchronization mechanism. The three core mechanisms of the synchronization hypothesis, nevertheless, may suggest specific neural substrates that are needed for the articulation of the syllable.

Target approximation, as discussed in Section 3 (cf. in particular the graphic illustration in Figures 4 and 6), would imply that the neural commands sent to the articulatory muscles are in the form of underlying targets rather than surface trajectories. Only in this way can the contextual variability in surface acoustic trajectories due to physical laws, mainly inertia, be articulatorily generated. Target approximation points out a clear forward relation from articulation to acoustics, which can be learned through analysis by synthesis (Cohen \& 't Hart, 1967). In this process, the articulatory system would repeatedly generate surface trajectories until a best fit is found, and the articulatory manoeuvre that can generate the best fit is stored as the learned target, as has been computationally simulated (Prom-on, Birkholz \& Xu, 2014; $\mathrm{Xu} \&$ Prom-on, 2014; Xu et al., 2019). Analysis by synthesis requires that continuous acoustic signals remain available during learning, to serve as auditory templates for imitative learning (Kuhl, 1989; Margoliash \& Schmidt, 2010). Both articulatory targets and surface acoustics therefore need to be neurally represented.

For edge synchronization, two critical neural mechanisms need to be in place. Firstly, there should be centrally generated signals for initiating each syllable. These signals, probably in the form of neural pulses, cannot be periodic, however, because their intervals need to frequently change with linguistic and paralinguistic factors such as stress (Fry, 1958), position in word and phrase (Nakatani, O'connor \& Aston, 1981), prosodic focus (de Jong, 2004; Xu, 1999; Xu \& $\mathrm{Xu}, 2005)$ and speaking style (Baker \& Bradlow, 2009). Secondly, there should be neural mechanisms to coactivate all the involved articulators without significant time delay or discrepancy. One way to achieve this is to bring the neural control areas close to each other in the brain to ensure quick communication. This may indeed have happened during the evolution of the human brain. Belyk (2017:180) suggests that an evolutionary reorganization has brought expiration, phonation and articulation into proximity in the brain, creating a small-world architecture (Sporns, 2006; Sporns \& Zwi, 2004) that would function efficiently. It would be interesting to examine in future research whether and how exactly this small-world architecture enables the synchronization of laryngeal, supralaryngeal, consonantal and vocalic articulations.

For tactile anchoring, as discussed in 3.3, the precision of synchronization depends on the quality of sensorimotor feedback (Mechsner et al., 2001). There should therefore be sensorimotor pathways that enable effective feedback control. The critical role of timing control has been recently demonstrated in songbirds by Kubikova et al. (2014) and Tanakaa et al. (2016), showing that disorders like stuttering can be induced in Zebra finch by modifying the gene critical for timing control. Interestingly, the induced changes did not affect the structure of individual syllables in the bird songs. This is consistent with the synchronization hypothesis in which tactile anchoring and target approximation are two separate mechanisms, the former relying crucially on feedback control, while the latter relying mainly on feedforward control (Perkell, 2012; Prom-on et al., 2009).

Even with the right genetic disposition, not only the ability to control the key articulators, but also the pathway to the brainstem may need time to fully develop after birth before 
synchronization can be attempted. This could be why canonical babbling, and with it the ability to produce syllables, start to emerge only around 6 months after birth (Kuhl \& Meltzoff, 1996), and even the order of the appearance of lingual consonants involved in the babbling follows that of the development of the tactile receptors in the tongue (Lyubimova et al., 1999).

\section{Conclusions}

This paper has proposed a new hypothesis about the syllable, positing it as a synchronization mechanism that makes the central nervous control of multiple articulatory movements in speech production possible by eliminating most of the temporal degrees of freedom. The hypothesis postulates three specific mechanisms: target approximation, edge synchronization and tactile anchoring, which work together to execute the syllable-a recurring synchronization of multiple articulatory movements.

Despite the evidence cited, the synchronization hypothesis is highly tentative. But it is more explicit than previous models of the syllable. This makes the hypothesis readily falsifiable. Both the general premise and the component mechanisms can be challenged by further research. The general premise based on degrees of freedom can be contested by modeling experiments that show clear advantages of flexible timing over synchronized timing in motor learning. Target approximation can be countered by alternative models that can better explain and predict finedetailed continuous articulatory and acoustic patterns. Edge synchronization can be refuted by clear demonstration of systematic misalignment of the target approximation movements of $\mathrm{C}$, $\mathrm{V}, \mathrm{F}_{0}$ or phonation at syllable onset and offset. And tactile anchoring can be contradicted or weakened by demonstration that articulation is little affected by reduction of tactile feedback.

\section{Acknowledgements}

This work was supported in part by the National Institutes of Health (NIH) Grant No. 1R01DC03902 and the Leverhulme Trust Research Project Grant RPG-2019-241. The funders had no role in study design, data collection and analysis, decision to publish, or preparation of the manuscript. I would also like to thank Fang Liu, Hong Gao, Anqi Xu, Santitham Prom-on and Peter Birkholz for their contribution to the development of this work. 


\section{References}

Abramson, A. S. (1978). Static and dynamic acoustic cues in distinctive tones. Language and Speech 21(4), 319-325.

Adler, R. (1946). A study of locking phenomena in oscillators. Proceedings of the IRE 34(6), 351-357.

Anderson-Hsieh, J., Riney, T. \& Koehler, K. (1994). Connected speech modifications in the English of Japanese ESL learners. Ideal 7, 31-52.

Arvaniti, A. \& Ladd, D. R. (2015). Underspecification in intonation revisited: a reply to Xu, Lee, Prom-on and Liu. Phonology 32, 537-541.

Arvaniti, A., Ladd, D. R. \& Mennen, I. (1998). Stability of tonal alignment: the case of Greek prenuclear accents. Journal of Phonetics 36, 3-25.

Atterer, M. \& Ladd, D. R. (2004). On the phonetics and phonology of "segmental anchoring" of F0: Evidence from German. Journal of Phonetics 32, 177-197.

Bailly, G. \& Holm, B. (2005). SFC: a trainable prosodic model. Speech Communication 46, 348-364.

Baker, R. E. \& Bradlow, A. R. (2009). Variability in word duration as a function of probability, speech style, and prosody. Language and speech 52(4), 391-413.

Baldissera, F., Cavallari, P., Marini, G. \& Tassone, G. (1991). Differential control of in-phase and anti-phase coupling of rhythmic movements of ipsilateral hand and foot. Experimental Brain Research 83, 375-380.

Barbosa, P. \& Bailly, G. (1994). Characterisation of rhythmic patterns for text-to-speech synthesis. Speech Communication 15(1-2), 127-137.

Bell-Berti, F. \& Krakow, R. A. (1991). Anticipatory velar lowering: A coproduction account. Journal of the Acoustical Society of America 90, 112-123.

Belyk, M. \& Brown, S. (2017). The origins of the vocal brain in humans. Neuroscience \& Biobehavioral Reviews 77, 177-193.

Bennett, M., Schatz, M. F., Rockwood, H. \& Wiesenfeld, K. (2002). Huygens's clocks. Proceedings: Mathematics, Physical and Engineering Sciences, 563-579.

Benoit, C. (1986). Note on the use of correlation in speech timing. Journal of the Acoustical Society of America 80, 1846-1849.

Bernstein, N. A. (1967). The co-ordination and regulation of movements. Oxford: Pergamon Press.

Bertoncini, J. \& Mehler, J. (1981). Syllables as units in infant speech perception. Infant Behavior and Development 4(0), 247-260.

Birkholz, P. \& Jackel, D. (2003). A three-dimensional model of the vocal tract for speech synthesis. In Proceedings of The 15th International Congress of Phonetic Sciences, Barcelona, Spain, 2597-2600. 
Birkholz, P., Kroger, B. J. \& Neuschaefer-Rube, C. (2011). Model-Based Reproduction of Articulatory Trajectories for Consonant-Vowel Sequences. Audio, Speech, and Language Processing, IEEE Transactions on 19, 1422-1433.

Bladon, R. A. W. \& Al-Bamerni, A. (1976). Coarticulation resistance of English /1/. Journal of Phonetics 4, 135-150.

Blevins, J. (2001). The syllable in phonological theory. In Handbook of Phonological Theory. J. Goldsmith. Cambridge, MA: Blackwell. pp. 206-244.

Blevins, J. (2003). Evolutionary phonology: The emergence of sound patterns. Cambridge: Cambridge University Press.

Bolinger, D. (1961). Contrastive accent and contrastive stress. Language 37, 83-96.

Boyce, S. E., Krakow, R. A. \& Bell-Berti, F. (1991). Phonological underspecification and speech motor organization. Phonology 8, 210-236.

Browman, C. P. \& Goldstein, L. (1988). Some notes on syllable structure in articulatory phonology. Phonetica 45(2-4), 140-155.

Browman, C. P. \& Goldstein, L. (1989). Articulatory gestures as phonological units. Phonology 6, 201-251.

Browman, C. P. \& Goldstein, L. (1992a). Articulatory phonology: An overview. Phonetica 49, 155-180.

Browman, C. P. \& Goldstein, L. (1992b). Targetless schwa: an articulatory analysis. In Papers in Laboratory Phonology II: Gesture, segment, prosody. G. J. Docherty and R. Ladd. Cambridge: Cambridge University Press. pp. 26-36.

Browman, C. P. \& Goldstein, L. M. (2000). Competing constraints on intergestural coordination and self-organization of phonological structures. Les Cahiers de l'ICP, Bulletin de la Communication Parlée 5, 25-34.

Buchanan, J. J. \& Ryu, Y. U. (2005). The interaction of tactile information and movement amplitude in a multijoint bimanual circle-tracing task: Phase transitions and loss of stability. The Quarterly Journal of Experimental Psychology Section A 58(5), 769-787.

Byrd, D. (1995). C-centers revisited. Phonetica 52(4), 285-306.

Byrd, D., Kaun, A., Narayanan, S. \& Saltzman, E. (2000). Phrasal signatures in articulation. In Papers in Laboratory Phonology V: Acquisition and the Lexicon. M. B. Broe and J. B. Pierrehumbert. Cambridge: Cambridge University Press. pp. 70-87.

Byrd, D. \& Saltzman, E. (2003). The elastic phrase: Modeling the dynamics of boundaryadjacent lengthening. Journal of Phonetics 31, 149-180.

Caspers, J. \& van Heuven, V. J. (1993). Effects of time pressure on the phonetic realization of the Dutch accent-lending pitch rise and fall. Phonetica 50, 161-171.

Chao, Y. R. (1968). A Grammar of Spoken Chinese. Berkeley, CA: University of California Press.

Chen, Y. \& Xu, Y. (2006). Production of weak elements in speech -- Evidence from f0 patterns of neutral tone in standard Chinese. Phonetica 63, 47-75. 
Cheng, C. \& Xu, Y. (2013). Articulatory limit and extreme segmental reduction in Taiwan Mandarin. Journal of the Acoustical Society of America 134(6), 4481-4495.

Chiosáin, M. N., Welby, P. \& Espesser, R. (2012). Is the syllabification of Irish a typological exception? An experimental study. Speech Communication 54(1), 68-91.

Chiu, F., Fromont, L., Lee, A. \& Xu, Y. (2015). Long-distance anticipatory vowel-to-vowel assimilatory effects in French and Japanese. In Proceedings of The 18th International Congress of Phonetic Sciences, Glasgow, UK: 1008-1012.

Clements, G. N. (1976). Vowel harmony in nonlinear generative phonology. Bloomington: Indiana University Linguistics Club.

Clements, G. N. \& Keyser, S. J. (1983). CV phonology. a generative theory of the syllable. Linguistic Inquiry Monographs Cambridge, Mass.(9), 1-191.

Cohen, A. and 't Hart, J. (1967). On the anatomy of intonation. Lingua 19: 177-192.

Content, A., Kearns, R. K. \& Frauenfelder, U. H. (2001). Boundaries versus onsets in syllabic segmentation. Journal of Memory and Language 45(2), 177-199.

Cooper, F. S., Delattre, P. C., Liberman, A. M., Borst, J. M. \& Gerstman, L. J. (1952). Some experiments on the perception of synthetic speech sounds. Journal of the Acoustical Society of America 24, 597-606.

Cooper, F. S., Liberman, A. M. \& Borst, J. M. (1951). The interconversion of audible and visible patterns as a basis for research in the perception of speech. Proceedings of the National Academy of Sciences 37, 318-325.

Cowie, R. I. and Douglas-Cowie, E. (1983). Speech production in profound postlingual deafness. In Hearing science and hearing disorders: Elsevier pp. 183-230.

Cummins, F. (2011). Periodic and Aperiodic Synchronization in Skilled Action. Frontiers in Human Neuroscience 5(170).

Cummins, F., Li, C. \& Wang, B. (2013). Coupling among speakers during synchronous speaking in English and Mandarin. Journal of Phonetics 41(6), 432-441.

Cummins, F. \& Port, R. (1998). Rhythmic constraints on stress timing in English. Journal of Phonetics 26, 145-171.

Cutler, A., Mehler, J., Norris, D. \& Segui, J. (1986). The syllable's differing role in the segmentation of French and English. Journal of Memory and Language 25(4), 385-400.

D'Imperio, M. (2001). Focus and tonal structure in Neapolitan Italian. Speech Communication 33, 339-356.

DeFrancis, J. F. (1989). Visible Speech: The Diverse Oneness of Writing Systems. Honolulu: University of Hawaii Press.

de Jong, K. (2001). Rate-induced resyllabification revisited. Language and Speech 44, 197216.

de Jong, K. (2004). Stress, lexical focus, and segmental focus in English: patterns of variation in vowel duration. Journal of Phonetics 32, 493-516. 
Delattre, P. C., Liberman, A. M. \& Cooper, F. S. (1955). Acoustic Loci and Transitional Cues for Consonants. Journal of the Acoustical Society of America 27(4), 769-773.

Dell, G. S. (1988). The retrieval of phonological forms in production: Tests of predictions from a connectionist model. Journal of Memory and Language 27, 124-142.

Dembowski, J., Lindstrom, M. J. \& Westbury, J. R. (1998). Articulator point variability in the production of stop consonants. In Neuromotor speech disorders: nature, assessment, and management. M. P. Cannito, K. M. Yorkston and D. R. Beukelman. Baltimore: Paul H. Brookes. pp. 27-46.

Duanmu, S. (2009). Syllable structure: The limits of variation: Oxford University Press.

Easton, T. A. (1972). On the normal use of reflexes: The hypothesis that reflexes form the basic language of the motor program permits simple, flexible specifications of voluntary movements and allows fruitful speculation. American Scientist 60(5), 591-599.

Edwards, J. R., Beckman, M. E. \& Fletcher, J. (1991). The articulatory kinematics of final lengthening. Journal of the Acoustical Society of America 89, 369-382.

Eriksson, A. (2012). Aural/acoustic vs. automatic methods in forensic phonetic case work. In Forensic Speaker Recognition: Law Enforcement and Counter-Terrorism. A. Neustein and H. A. Patil. New York: Springer. pp. 41-69.

Farnetani, E. and Recasens, D. (1997). Coarticulation and connected speech processes. The handbook of phonetic sciences: 371-404.

Fitch, W. T. (2010). The evolution of language: Cambridge University Press.

Fowler, C. A. (1980). Coarticulation and theories of extrinsic timing. Journal of Phonetics 8, 113-133.

Fowler, C. A. (1981). Production and perception of coarticulation among stressed and unstressed vowels. Journal of Speech and Hearing Research 46, 127-139.

Fowler, C. A. (1994). Invariants, specifiers, cues: An investigation of locus equations as information for place of articulation. Perception and Psychophysics 55, 597-610.

Fowler, C. A., Rubin, P., Remez, R. E. and Turvey, M. T. (1980). Implications for speech production of a general theory of action. In Language Production. Butterworth. New York: Academic Press pp. 373-420.

Frota, S. (2002). Tonal association and target alignment in European Portuguese nuclear falls. In Laboratory Phonology VII. C. Gussenhoven and N. Warner. Berlin: Mouton de Gruyter. pp. 387-418.

Fry, D. B. (1958). Experiments in the perception of stress. Language and Speech 1, 126-152.

Fudge, E. (1969). Syllables. Journal of Linguistics 5: 253-86.

Fujimura, O. (1994). C/D Model: A computational model of phonetic implementation. In Language and Computations. E. S. Ristad. Providence, RI: American Math Society. pp. $1-20$. 
Fujisaki, H. (1983). Dynamic characteristics of voice fundamental frequency in speech and singing. In The Production of Speech. P. F. MacNeilage. New York: Springer-Verlag. pp. 39-55.

Fujisaki, H., Wang, C., Ohno, S. \& Gu, W. (2005). Analysis and synthesis of fundamental frequency contours of Standard Chinese using the command-response model. Speech communication 47, 59-70.

Gafos, A. I. \& Benus, S. (2006). Dynamics of phonological cognition. Cognitive science 30(5), 905-943.

Gandour, J., Potisuk, S. \& Dechongkit, S. (1994). Tonal coarticulation in Thai. Journal of Phonetics 22, 477-492.

Gao, H. \& Xu, Y. (2010). Ambisyllabicity in English: How real is it? In Proceedings of The 9th Phonetics Conference of China (PCC2010), Tianjin.

Gay, T. (1968). Effect of speaking rate on diphthong formant movements. Journal of the Acoustical Society of America 44, 1570-1573.

Gay, T. (1978). Articulatory units: segments or syllables. Syllables and segments, 121-132.

Gick, B. (2003). Articulatory correlates of ambisyllabicity in English glides and liquids. In Papers in Laboratory Phonology VI: Constraints on Phonetic Interpretation. J. Local, R. Ogden and R. Temple. Cambridge: Cambridge University Press. pp. 222-236.

Gimson, A. C. (1970). An introduction to the pronunciation of English. London: Arnold.

Gnanadesikan, A. E. (2011). The writing revolution: Cuneiform to the internet: John Wiley \& Sons.

Goldstein, L., Byrd, D. \& Saltzman, E. (2006). The role of vocal tract gestural action units in understanding the evolution of phonology. Action to language via the mirror neuron system, 215-249.

Goslin, J. \& Frauenfelder, U. H. (2001). A Comparison of Theoretical and Human Syllabification. Language and Speech 44(4), 409-436.

Grosvald, M. (2010). Long-Distance Coarticulation in Spoken and Signed Language: An Overview. Language and Linguistics Compass 4(6): 348-362.

Gu, W. \& Lee, T. (2007). Effects of tonal context and focus on Cantonese F0. In Proceedings of The 16th International Congress of Phonetic Sciences, Saarbrucken: 1033-1036.

Haken, H., Kelso, J. A. S. \& Bunz, H. (1985). A Theoretical Model of Phase Transitions in Human Hand Movements. Biological Cybernetics 51, 347-356.

Heid, S. and Hawkins, S. (2000). An acoustical study of long-domain/r/and/1/coarticulation. In Proceedings of Proceedings of the 5th seminar on speech production: Models and data. Citeseer: 77-80.

Hieke, A. E. (1984). Linking as a marker of fluent speech. Language and Speech 27(4), 343354.

Hoard, J. E. (1971). Aspiration, tenseness, and syllabication in English. Language: 133-140. 
Hockett, C. F. (1955). A manual of phonology (International Journal of American Linguistics, Memoir 11). Baltimore: Waverly Press.

Hooper, J. B. (1972). The syllable in phonological theory. Language 48, 525-540.

hubpages.com (2014). How to Hit a Great Smash in Badminton. Accessed 9 September 8, 2016 from http://hubpages.com/games-hobbies/Badminton-Smash-How-to-Play-the-Shot\# (courtesy of Michael Hayes at HowTheyPlay.com)

Huffman, M. K. (1987). Measures of phonation type in Hmong. The Journal of the Acoustical Society of America 81(2): 495-504.

Huygens, C. (1893). a letter to his father, dated 26 Feb. 1665. In Ouevres Completes de Christian Huyghens. M. Nijhoff. The Hague, The Netherlands: Societe Hollandaise des Sciences. 5, pp. 243.

Huys, R. (2010). The Dynamical Organization of Limb Movements. In Nonlinear dynamics in human behavior: Springer. pp. 69-90.

Iskarous, K., Fowler, C. A. \& Whalen, D. H. (2010). Locus equations are an acoustic expression of articulator synergy. The Journal of the Acoustical Society of America 128(4), 20212032.

Jakobson, R., Fant, C. G. \& Halle, M. (1951). Preliminaries to Speech Analysis. The distinctive features and their correlates. Cambridge, MA: MIT Press.

Johansson, R. S. \& Flanagan, J. R. (2009). Coding and use of tactile signals from the fingertips in object manipulation tasks. Nature Reviews Neuroscience 10(5), 345.

Kay, B., Kelso, J., Saltzman, E. \& Schöner, G. (1987). Space-time behavior of single and bimanual rhythmical movements: Data and limit cycle model. Journal of Experimental Psychology: Human Perception and Performance 13(2), 178.

Keating, P. A. (1988). Underspecification in phonetics. Phonology 5, 275-292.

Kelso, J. A. S. (1984). Phase transitions and critical behavior in human bimanual coordination. American Journal of Physiology: Regulatory, Integrative and Comparative 246, R1000R1004.

Kelso, J. A. S., Saltzman, E. L. \& Tuller, B. (1986). The dynamical perspective on speech production: data and theory. Journal of Phonetics 14, 29-59.

Kelso, J. A. S., Southard, D. L. \& Goodman, D. (1979). On the nature of human interlimb coordination. Science 203, 1029-1031.

Kelso, J. A. S., Tuller, B. \& Harris, K. S. (1983). A "dynamic pattern" perspective on the control and coordination of movement. In The Production of Speech. P. F. MacNeilage. New York: Springer-Verlag. pp. 137-173.

Kelso, S. J. A., Fink, P. W., DeLaplain, C. R. \& Carson, R. G. (2001). Haptic information stabilizes and destabilizes coordination dynamics. Proceedings of the Royal Society of London B: Biological Sciences 268(1472), 1207-1213.

Kent, R. \& Minifie, F. (1977). Coarticulation in recent speech production models. Journal of Phonetics 5, 115-133. 
Kent, R. D. \& Moll, K. L. (1972). Tongue Body Articulation during Vowel and Diphthong Gestures. Folia phoniat 24, 278-300.

Kingston, J. (2019). The interface between phonetics and phonology. In The Routledge Handbook of Phonetics. W. F. Katz and P. F. Assmann. New York: Routledge. pp. 359400.

Koh, K., Kwon, H. J., Yoon, B. C., Cho, Y., Shin, J.-H., Hahn, J.-O., Miller, R. H., Kim, Y. H. \& Shim, J. K. (2015). The role of tactile sensation in online and offline hierarchical control of multi-finger force synergy. Experimental Brain Research 233(9), 2539-2548.

Kohler, K. J. (1966). Is the syllable a phonological universal? Journal of Linguistics 2(02), 207208.

Kozhevnikov, V. A. \& Chistovich, L. A. (1965). Speech: Articulation and Perception. Washington, DC: Translation by Joint Publications Research Service. JPRS 30543.

Kubikova, L., Bosikova, E., Cvikova, M., Lukacova, K., Scharff, C. \& Jarvis, E. D. (2014). Basal ganglia function, stuttering, sequencing, and repair in adult songbirds. Scientific Reports 4, 6590.

Kuhl, P. K. (1989). On babies, birds, modules, and mechanisms: A comparative approach to the acquisition of vocal communication. In The comparative psychology of audition: Perceiving complex sounds. R. J. Dooling and S. H. Hulse. Hillsdale, NJ: Erlbaum. pp. 379-419.

Kuhl, P. K. \& Meltzoff, A. N. (1996). Infant vocalizations in response to speech: Vocal imitation and developmental change. The Journal of the Acoustical Society of America $100(4), 2425-2438$.

Kühnert, B. \& Nolan, F. (1999). The origin of coarticulation. In Coarticulation: Theory, Data and Techniques. W. J. Hardcastle and N. Newlett. Cambridge: Cambridge University Press. pp. 7-30.

Laboissiere, R., Ostry, D. J. \& Feldman, A. G. (1996). The control of multi-muscle systems: human jaw and hyoid movements. Biological Cybernetics 74, 373-384.

Labrune, L. (2012). Questioning the universality of the syllable: evidence from Japanese. Phonology 29(01), 113-152.

Ladd, D. R., Faulkner, D., Faulkner, H. \& Schepman, A. (1999). Constant "segmental anchoring" of F0 movements under changes in speech rate. Journal of the Acoustical Society of America 106, 1543-1554.

Ladd, D. R., Mennen, I. \& Schepman, A. (2000). Phonological conditioning of peak alignment in rising pitch accents in Dutch. Journal of the Acoustical Society of America 107, 26852696.

Ladefoged, P. (1982). A Course in Phonetics. New York: Hartcourt Brace Jovanovich.

Lane, H. and Webster, J. W. (1991). Speech deterioration in postlingually deafened adults. The Journal of the Acoustical Society of America 89(2): 859-866.

Laniran, Y. O. \& Clements, G. N. (2003). Downstep and high raising: interacting factors in Yoruba tone production. Journal of Phonetics 31, 203-250. 
Latash, M. L., Scholz, J. P. \& Schöner, G. (2007). Toward a new theory of motor synergies. Motor control 11(3), 276-308.

Lee, A., Xu, Y. and Prom-On, S. (2017). Pre-low raising in Japanese pitch accent. Phonetica 74: 231-246.

Lehiste, I. (1960). An acoustic-phonetic study of internal open juncture. Phonetica 5(Suppl. 1), 5-54.

Levelt, W. J. M., Roelofs, A. \& Meyer, A. S. (1999). A theory of lexical access in speech production. Behavioral and brain sciences 22(1), 1-38.

Liberman, I. Y., Shankweiler, D., Fischer, F. W. and Carter, B. (1974). Explicit syllable and phoneme segmentation in the young child. Journal of experimental child psychology 18(2): 201-212.

Liberman, A. M., Cooper, F. S., Shankweiler, D. P. \& Studdert-Kennedy, M. G. (1967). Perception of the speech code. Psychological Review 74, 431-461.

Lindblom, B. (1963). Spectrographic study of vowel reduction. Journal of the Acoustical Society of America 35, 1773-1781.

Lindblom, B. \& Sussman, H. M. (2012). Dissecting coarticulation: How locus equations happen. Journal of Phonetics 40(1), 1-19.

Ling, B. \& Liang, J. (2015). Tonal alignment in shanghai Chinese. In Proceedings of COCOSDA2015, 128-132.

Liu, F., Xu, Y., Prom-on, S. \& Yu, A. C. L. (2013). Morpheme-like prosodic functions: Evidence from acoustic analysis and computational modeling. Journal of Speech Sciences 3(1), 85-140.

Locke, J. L. (1983). Phonological Acquisition and Change. London: Academic Press.

Löfqvist, A. (1991). Proportional timing in speech motor control. Journal of Phonetics 19, 343350 .

Lyubimova, Z. V., Sisengalieva, G. Z., Chulkova, N. Y., Smykova, O. I. \& Selin, S. V. (1999). Role of tactile receptor structures of the tongue in speech sound production of infants of the first year of life. Bulletin of Experimental Biology and Medicine 127(2), 115-119.

MacNeilage, P. F. (1998). The frame/content theory of evolution of speech production. Behavioral and Brain Sciences 21, 499-546.

Magen, H. S. (1997). The extent of vowel-to-vowel coarticulation in English. Journal of Phonetics 25: 187-205.

Margoliash, D. \& Schmidt, M. F. (2010). Sleep, off-line processing, and vocal learning. Brain and Language 115(1), 45-58.

Mechsner, F., Kerzel, D., Knoblich, G. \& Prinz, W. (2001). Perceptual basis of bimanual coordination. Nature 414, 69-73. 
Mechsner, F., Stenneken, P., Cole, J., Aschersleben, G. \& Prinz, W. (2007). Bimanual circling in deafferented patients: evidence for a role of visual forward models. journal of Neuralphsiology 1, 259-282.

Menzerath, P. \& de Lacerda, A. (1933). Koartikulation, Seuerung und Lautabgrenzung. Berlin and Bonn: Fred. Dummlers.

Moll, K. \& Daniloff, R. (1971). Investigation of the timing of velar movement during speech. Journal of the Acoustical Society of America 50, 678-684.

Moon, S.-J. \& Lindblom, B. (1994). Interaction between duration, context, and speaking style in English stressed vowels. Journal of the Acoustical Society of America 96, 40-55.

Munhall, K. G. (1985). An examination of intra-articulator relative timing. Journal of the Acoustical Society of America 78, 1548-1553.

Myers, S. (1998). Surface underspecification of tone in Chichewa. Phonology 15, 367-392.

Nakatani, L. H., O'connor, K. D. \& Aston, C. H. (1981). Prosodic aspects of American English speech rhythm. Phonetica 38, 84-106.

Nam, H., Goldstein, L. \& Saltzman, E. (2009). Self-organization of syllable structure: A coupled oscillator model. In Approaches to phonological complexity. F. Pellegrino, E. Marsico, I. Chitoran and C. Coupé. New York: Mouton de Gruyter. pp. 299-328.

Nguyen, N. and Fagyal, Z. (2008). Acoustic aspects of vowel harmony in French. Journal of Phonetics 36(1): 1-27.

Nolan, F. \& Asu, E. L. (2009). The Pairwise Variability Index and Coexisting Rhythms in Language. Phonetica 66(1-2), 64-77.

Ohala, J. J. (1992). Alternatives to the sonority hierarchy for explaining segmental sequential constraints. In Papers from the Parasession on the Syllable. Chicago: Chicago Linguistic Society. pp. 319-338.

Ohala, J. J. (1994). Towards a universal, phonetically-based, theory of vowel harmony. In Proceedings of Third International Conference on Spoken Language Processing, Yokohama, 491-494.

Ohala, J. J. \& Kawasaki, H. (1984). Prosodic phonology and phonetics. Phonology 1, 113-127.

Öhman, S. E. G. (1966). Coarticulation in VCV utterances: Spectrographic measurements. Journal of the Acoustical Society of America 39, 151-168.

Ostry, D. J., Gribble, P. L. \& Gracco, V. L. (1996). Coarticulation of jaw movements in speech production: is context sensitivity in speech kinematics centrally planned? Journal of Neurosicence 16, 1570-1979.

Perkell, J. S. (2012). Movement goals and feedback and feedforward control mechanisms in speech production. Journal of Neurolinguistics 25(5), 382-407.

Peterson, G. E. \& Lehiste, I. (1960). Duration of syllable nuclei in English. Journal of the Acoustical Society of America 32, 693-703.

Peterson, G. E., Wang, W. S. Y. and Sivertsen, E. (1958). Segmentation techniques in speech synthesis. The Journal of the Acoustical Society of America 30(8): 739-742. 
Pierrehumbert, J. (1980). The Phonology and Phonetics of English Intonation. Ph.D. dissertation, MIT, Cambridge, MA. [Published in 1987 by Indiana University Linguistics Club, Bloomington].

Pikovsky, A., Rosenblum, M. \& Kurths, J. (2001). Synchronization -- A universal concept in nonlinear sciences: Cambridge University Press.

Pinker, S. (1995). The language instinct: The new science of language and mind: Penguin UK.

Prieto, P. \& Torreira, F. (2007). The segmental anchoring hypothesis revisited: Syllable structure and speech rate effects on peak timing in Spanish. Journal of Phonetics 35(4), 473-500.

Prom-on, S., Birkholz, P. \& Xu, Y. (2013). Training an articulatory synthesizer with continuous acoustic data. In Proceedings of Interspeech 2013, 349-353.

Prom-on, S., Birkholz, P. \& Xu, Y. (2014). Identifying underlying articulatory targets of Thai vowels from acoustic data based on an analysis-by-synthesis approach. EURASIP Journal on Audio, Speech, and Music Processing 23. https://doi.org/10.1186/16874722-2014-23

Prom-on, S., Xu, Y. \& Thipakorn, B. (2009). Modeling tone and intonation in Mandarin and English as a process of target approximation. Journal of the Acoustical Society of America 125, 405-424.

Pulgram, E. (1970). Syllable, word, nexus, cursus. The Hague: Mouton.

Recasens, D. (1984a). Vowel-to-vowel coarticulation in Catalan VCV sequences. Journal of the Acoustical Society of America 76, 1624-1635.

Recasens, D. (1984b). V-to-C coarticulation in Catalan VCV sequences: An articulatory and acoustical study. Journal of Phonetics 12, 61-73.

Ridderikhoff, A., Peper, C. E. \& Beek, P. J. (2007). Error correction in bimanual coordination benefits from bilateral muscle activity: evidence from kinesthetic tracking. Experimental Brain Research 181, 31-48.

Ringel, R. L. \& Ewanowski, S. J. (1965). Oral Perception: 1. Two-Point Discrimination. Journal of Speech, Language, and Hearing Research 8(4), 389-398.

Sadat-Tehrani, N. (2009). The alignment of $\mathrm{L}+\mathrm{H}^{*}$ pitch accents in Persian intonation. Journal of the International Phonetic Association 39, 205-230.

Saitou, T., Unoki, M. \& Akagi, M. (2005). Development of an F0 control model based on F0 dynamic characteristics for singing-voice synthesis. Speech Communication 46(3-4), 405-417.

Saltzman, E. L. \& Munhall, K. G. (1989). A dynamical approach to gestural patterning in speech production. Ecological Psychology 1, 333-382.

Saltzman, E., Nam, H., Krivokapić, J. \& Goldstein, L. (2008). A task-dynamic toolkit for modeling the effects of prosodic structure on articulation. In Proceedings of Speech Prosody 2008, 175-184. 
Schiller, N. O., Meyer, A. S. \& Levelt, W. J. (1997). The syllabic structure of spoken words: Evidence from the syllabification of intervocalic consonants. Language and Speech 40(2), 103-140.

Schmidt, R. C., Carello, C. \& Turvey, M. T. (1990). Phase transitions and critical fluctuations in the visual coordination of rhythmic movements between people. Journal of Experimental Psychology: Human Perception and Performance 16, 227-247.

Selkirk, E. O. (1982). The syllable. In The structure of phonological representations, part II. H. v. d. Hulst and N. Smith. Dordrecht: Foris. pp. 337-383.

Semjen, A. \& Ivry, R. B. (2001). The coupled oscillator model of between-hand coordination in alternate-hand tapping: a reappraisal. Journal of Experimental Psychology: Human Perception and Performance 27(2), 251.

Shattuck-Hufnagel, S. (2011). The role of the syllable in speech production in American English: A fresh consideration of the evidence. In Handbook of the Syllable. C. E. Cairns and E. Raimy. Boston: Brill. pp. 197-224.

Spencer, R. M. C., Ivry, R. B., Cattaert, D. \& Semjen, A. (2005). Bimanual coordination during rhythmic movements in the absence of somatosensory feedback. journal of Neuralphsiology 94, 2901-2910.

Sporns, O. (2006). Small-world connectivity, motif composition, and complexity of fractal neuronal connections. Biosystems 85(1), 55-64.

Sporns, O. \& Zwi, J. D. (2004). The small world of the cerebral cortex. Neuroinformatics 2(2), $145-162$.

Sproat, R. \& Fujimura, O. (1993). Allophonic variation in English /1/ and its implications for phonetic implementation. Journal of Phonetics 21, 291-311.

Steriade, D. (1982). Greek prosodies and the nature of syllabification, Massachusetts Institute of Technology.

Steriade, D. (1995). Underspecification and markedness. In Handbook of Phonological Theory. J. A. Goldsmith. Oxford: Basil Blackweell. pp. 114-174.

Steriade, D. (1999). Alternatives to syllable-based accounts of consonantal phonotactics. In Proceedings of linguistics and phonetics 1998: Item order in language and speech. $\mathrm{O}$. Fujimura, B. D. Joseph and B. Palek. Prague: Karolinum Press. pp. 205-245.

Stetson, R. H. (1951). Motor Phonetics: A study of Speech Movements in Action. Amsterdam: North Holland.

Stevens, K. N. (1998). Acoustic Phonetics. Cambridge, MA: The MIT Press.

Stevens, K. N. \& Hanson, H. M. (2012). 12 Articulatory-Acoustic Relations as the Basis of Distinctive Contrasts. The Handbook of Phonetic Sciences 116, 424.

Tanakaa, M., Alvarado, J. S., Murugan, M. \& Mooney, R. (2016). Focal expression of mutant huntingtin in the songbird basal ganglia disrupts cortico-basal ganglia networks and vocal sequences. Proceedings of the National Academy of Sciences 113(12), E1720E1727. 
Taylor, P. (2009). Text-to-Speech Synthesis. Cambridge: Cambridge University Press.

Tiffany, W. R. (1980). The effects of syllable structure on diadochokinetic and reading rates. Journal of Speech and Hearing Research 23, 894-908.

Treiman, R. \& Danis, C. (1988). Syllabification of intervocalic consonants. Journal of Memory and Language 27, 87-104.

Turk, A., Nakai, S. \& Sugahara, M. (2006). Acoustic Segment Durations in Prosodic Research: A Practical Guide. In Methods in Empirical Prosody Research. S. Sudhoff, D. Lenertová, R. Meyeret al. Berlin, New York: De Gruyter. pp. 1-28.

Turvey, M. T. (1977). Preliminaries to a theory of action with reference to vision. Perceiving, acting and knowing, 211-265.

van Santen, J., Kain, A., Klabbers, E. \& Mishra, T. (2005). Synthesis of prosody using multilevel unit sequences. Speech Communication 46, 365-375.

Vennemann, T. (1988). Preference laws for syllable structure and the explanation of sound change. Berlin, Mouton de Gruyter.

Wayland, R. \& Jongman, A. (2003). Acoustic correlates of breathy and clear vowels: The case of Khmer. Journal of Phonetics 31(2), 181-201.

Wells, J. C. (1990). Syllabification and allophony. In S. Ramsaran. In Studies in the pronunciation of English: A commemorative volume in honour of A. C. Gimson. London: Routledge pp. 76-86.

West, P. (1999). The extent of coarticulation of English liquids: An acoustic and articulatory study. In Proceedings of Proc. 14th ICPhS: 1901-1904.

Whalen, D. H. (1990). Coarticulation is largely planned. Journal of Phonetics 18: 3-35.

Wilson, A. D., Bingham, G. P. \& Craig, J. C. (2003). Proprioceptive perception of phase variability. Journal of Experimental Psychology: Human Perception \& Performance 29, 1179-1190.

Wong, Y. W. (2006). Contextual Tonal Variations and Pitch Targets in Cantonese. In Proceedings of Speech Prosody 2006, Dresden, Germany, PS3-13-199.

Wong, Y. W. \& Xu, Y. (2007). Consonantal perturbation of f0 contours of Cantonese tones. In Proceedings of The 16th International Congress of Phonetic Sciences, Saarbrucken, 1293-1296.

Wood, S. A. J. (1996). Assimilation or coarticulation? Evidence from the temporal coordination of tongue gestures for the palatalization of Bulgarian alveolar stops. Journal of Phonetics 24, 139-164.

$\mathrm{Xu}$, A., Birkholz, P. \& Xu, Y. (2019). Coarticulation as synchronized dimension-specific sequential target approximation: An articulatory synthesis simulation. In Proceedings of The 19th International Congress of Phonetic Sciences, Melbourne, Australia

Xu, C. X. \& Xu, Y. (2003). Effects of consonant aspiration on Mandarin tones. Journal of the International Phonetic Association 33, 165-181. 
$\mathrm{Xu}, \mathrm{Y} .(1986)$. Acoustic-phonetic characteristics of junctures in Mandarin Chinese [in Chinese]. Zhongguo Yuwen [Journal of Chinese Linguistics](4), 353-360.

$\mathrm{Xu}, \mathrm{Y}$. (1997). Contextual tonal variations in Mandarin. Journal of Phonetics 25, 61-83.

$\mathrm{Xu}, \mathrm{Y}$. (1998). Consistency of tone-syllable alignment across different syllable structures and speaking rates. Phonetica 55, 179-203.

$\mathrm{Xu}, \mathrm{Y}$. (1999). Effects of tone and focus on the formation and alignment of F0 contours. Journal of Phonetics 27, 55-105.

Xu, Y. (2001). Fundamental frequency peak delay in Mandarin. Phonetica 58, 26-52.

$\mathrm{Xu}, \mathrm{Y}$. (2005). Speech melody as articulatorily implemented communicative functions. Speech Communication 46: 220-251.

$\mathrm{Xu}, \mathrm{Y}$. (2009). Timing and coordination in tone and intonation--An articulatory-functional perspective. Lingua 119(6), 906-927.

Xu, Y. \& Gao, H. (2018). FormantPro as a tool for speech analysis and segmentation. Revista de Estudos da Linguagem 26(4), 1435-1454.

$\mathrm{Xu}$, Y. \& Liu, F. (2006). Tonal alignment, syllable structure and coarticulation: Toward an integrated model. Italian Journal of Linguistics 18, 125-159.

Xu, Y. \& Liu, F. (2007). Determining the temporal interval of segments with the help of F0 contours. Journal of Phonetics 35, 398-420.

$\mathrm{Xu}, \mathrm{Y} . \&$ Prom-on, S. (2014). Toward invariant functional representations of variable surface fundamental frequency contours: Synthesizing speech melody via model-based stochastic learning. Speech Communication 57, 181-208.

$\mathrm{Xu}, \mathrm{Y} . \&$ Prom-on, S. (2015). Degrees of freedom in prosody modeling. In Speech Prosody in Speech Synthesis - Modeling, Realizing, Converting Prosody for High Quality and Flexible speech Synthesis. K. Hirose and J. Tao: Springer. pp. 19-34.

$\mathrm{Xu}, \mathrm{Y}$. and Prom-on, S. (2019). Economy of Effort or Maximum Rate of Information? Exploring Basic Principles of Articulatory Dynamics. Frontiers in Psychology 10(2469). DOI: 10.3389/fpsyg.2019.02469

$\mathrm{Xu}, \mathrm{Y}$. \& Wang, Q. E. (2001). Pitch targets and their realization: Evidence from Mandarin Chinese. Speech Communication 33, 319-337.

$\mathrm{Xu}, \mathrm{Y} . \& \mathrm{Xu}, \mathrm{C}$. (2005). Phonetic realization of focus in English declarative intonation. Journal of Phonetics 33(2), 159-197.

Yeou, M. (2004). Effects of focus, position and syllable structure on F0 alignment patterns in Arabic. In Proceedings of JEP-TALN 2004, Arabic Language Processing, Fez, 19-22. 